\title{
\begin{tabular}{l|l} 
Mibraries & DSpace@MIT
\end{tabular}
}

\author{
MIT Open Access Articles
}

\section{DISCOVERY OF THE THIRD TRANSIENT X-RAY BINARY IN THE GALACTIC GLOBULAR CLUSTER TERZAN 5}

The MIT Faculty has made this article openly available. Please share how this access benefits you. Your story matters.

Citation: Bahramian, Arash, Craig O. Heinke, Gregory R. Sivakoff, Diego Altamirano, Rudy Wijnands, Jeroen Homan, Manuel Linares, David Pooley, Nathalie Degenaar, and Jeanette C. Gladstone. “DISCOVERY OF THE THIRD TRANSIENT X-RAY BINARY IN THE GALACTIC GLOBULAR CLUSTER TERZAN 5." The Astrophysical Journal 780, no. 2 (December 17, 2013): 127. (C) 2014 The American Astronomical Society

As Published: http://dx.doi.org/10.1088/0004-637X/780/2/127

Publisher: IOP Publishing

Persistent URL: http://hdl.handle.net/1721.1/95440

Version: Final published version: final published article, as it appeared in a journal, conference proceedings, or other formally published context

Terms of Use: Article is made available in accordance with the publisher's policy and may be subject to US copyright law. Please refer to the publisher's site for terms of use. 


\title{
DISCOVERY OF THE THIRD TRANSIENT X-RAY BINARY IN THE GALACTIC GLOBULAR CLUSTER TERZAN 5
}

\author{
Arash Bahramian ${ }^{1}$, Craig O. Heinke ${ }^{1}$, Gregory R. Sivakoff ${ }^{1}$, Diego Altamirano ${ }^{2,3}$, Rudy Wijnands ${ }^{2}$, Jeroen Homan $^{4}$, \\ Manuel Linares $^{5,6}$, David Pooley ${ }^{7,8}$, Nathalie Degenaar ${ }^{9,10}$, and Jeanette C. Gladstone ${ }^{1}$ \\ ${ }^{1}$ Department of Physics, University of Alberta, CCIS 4-183, Edmonton, AB T6G 2E1, Canada; bahramia@ualberta.ca \\ ${ }^{2}$ Astronomical Institute "Anton Pannekoek," University of Amsterdam, Science Park 904, 1098 XH Amsterdam, The Netherlands \\ ${ }^{3}$ Physics \& Astronomy, University of Southampton, Southampton, Hampshire SO17 1BJ, UK \\ ${ }^{4}$ Kavli Institute for Astrophysics \& Space Research, Massachusetts Institute of Technology, 70 Vassar Street, Cambridge, MA 02139, USA \\ ${ }^{5}$ Instituto de Astrofísica de Canarias, c/Vía Láctea s/n, E-38205 La Laguna, Tenerife, Spain \\ ${ }^{6}$ Universidad de La Laguna, Department Astrofísica, E-38206 La Laguna, Tenerife, Spain \\ ${ }^{7}$ Department of Physics, Sam Houston State University, Huntsville, TX 77341, USA \\ ${ }^{8}$ Eureka Scientific, Inc., 2452 Delmer Street, Suite 100, Oakland, CA, 94602, USA \\ ${ }^{9}$ Department of Astronomy, University of Michigan, 500 Church Street, Ann Arbor, MI 48109, USA \\ Received 2013 August 21; accepted 2013 November 16; published 2013 December 17
}

\begin{abstract}
We report and study the outburst of a new transient X-ray binary (XRB) in Terzan 5, the third detected in this globular cluster, Swift J174805.3-244637 or Terzan 5 X-3. We find clear spectral hardening in Swift/XRT data during the outburst rise to the hard state, thanks to our early coverage (starting at $L_{X} \sim 4 \times 10^{34} \mathrm{erg} \mathrm{s}^{-1}$ ) of the outburst. This hardening appears to be due to the decline in relative strength of a soft thermal component from the surface of the neutron star (NS) during the rise. We identify a Type I X-ray burst in Swift/XRT data with a long (16 s) decay time, indicative of hydrogen burning on the surface of the NS. We use Swift/BAT, MAXI/GSC, Chandra/ACIS, and Swift/XRT data to study the spectral changes during the outburst, identifying a clear hard-tosoft state transition. We use a Chandra/ACIS observation during outburst to identify the transient's position. Seven archival Chandra/ACIS observations show evidence for variations in Terzan 5 X-3's nonthermal component but not the thermal component during quiescence. The inferred long-term time-averaged mass accretion rate, from the quiescent thermal luminosity, suggests that if this outburst is typical and only slow cooling processes are active in the NS core, such outbursts should recur every $\sim 10 \mathrm{yr}$.
\end{abstract}

Key words: binaries: close - globular clusters: individual (Terzan 5) - stars: neutron - X-rays: binaries - X-rays: bursts - X-rays: individual (Swift J174805.3-244637)

Online-only material: color figures

\section{INTRODUCTION}

Transient low-mass X-ray binaries (LMXBs) experience long periods (often years to tens of years) of quiescence. In quiescence matter flowing from the companion builds up in the accretion disk, punctuated by outbursts when the accretion disk crosses a pressure and temperature threshold, increases in viscosity, and dumps large quantities of matter onto the accreting compact object (e.g., see Lasota 2001, for a review). Their outbursts go through phases of varying X-ray spectra. These phases are generally interpreted as indicating the changing relative contributions of Comptonized optically thin emission versus blackbody-like emission from an accretion disk, as the accretion rate and geometry change (e.g., Remillard \& McClintock 2006; Done et al. 2007, for reviews). These X-ray spectral states have been studied both for black hole LMXBs, and for neutron star (NS) LMXBs (e.g., Hasinger \& van der Klis 1989; Gierliński \& Done 2002; Gilfanov et al. 2003; Gladstone et al. 2007; Lin et al. 2007), which show an additional component from the NS surface.

The spectra of NS LMXBs in quiescence $\left(L_{X} \lesssim 10^{33} \mathrm{erg} \mathrm{s}^{-1}\right)$ include thermal radiation from the (usually hydrogen) NS atmosphere (blackbody-like; Zavlin et al. 1996; Rajagopal \& Romani 1996), and often a harder nonthermal component, usually fit with a power-law (Campana et al. 1998). Several

\footnotetext{
${ }^{10}$ Hubble Fellow.
}

NS LMXBs have shown rapid, strong variability in quiescence indicative of accretion events, which can sometimes be clearly attributed to variation in both the thermal and power-law components (e.g., Rutledge et al. 2002; Campana et al. 2004; Cackett et al. 2010; Fridriksson et al. 2011). The thermal component can be produced either by re-radiation of stored heat from the cooling NS (Brown et al. 1998), or by lowlevel accretion (Zampieri et al. 1995; Deufel et al. 2001), which produce similar spectra (Zampieri et al. 1995).

Studies of the spectra of black hole LMXBs during their decline from the low/hard state into quiescence (as $L_{X}$ falls below $10^{35} \mathrm{erg} \mathrm{s}^{-1}$ ) have found clear softening (Corbel et al. 2006, 2008; Armas Padilla et al. 2013a; Plotkin et al. 2013). The softening of black hole LMXB spectra has been interpreted as a change in the origin of the X-ray emission, produced at low luminosities by either a radiatively inefficient hot flow (Esin et al. 1997; Gardner \& Done 2012) or synchrotron emission from a jet (Yuan \& Cui 2005; Pszota et al. 2008). A similar softening in the spectrum from the accretion flow occurs in NS systems at similar luminosities, where emission from the NS surface can play a role (Armas Padilla et al. 2011; Degenaar et al. 2013b; M. Linares et al. 2013).

Thermonuclear X-ray bursts burn accumulated hydrogen and/or helium on the NS surface, producing blackbody-like emission with a rapid rise, cooling over timescales of seconds to minutes (Lewin et al. 1993; Galloway et al. 2008). X-ray bursts occurring in hydrogen-poor environments (either due to 
no hydrogen being present in the accreted material, or hydrogen being stably burned during accretion) show different properties from those in hydrogen-rich environments. The ratio of energy released by fusion in a burst to energy released during accretion is lower for helium bursts compared to hydrogen bursts due to the lower energy available from fusion.

Helium bursts generally have faster rise and decline times, since hydrogen burning involves the CNO cycle and thus is limited by the speed of $\beta$-decays (Fujimoto et al. 1981). Pure He bursts can be ignited in NSs that accrete hydrogen at low mass accretion rates (e.g., Peng et al. 2007), but NSs known to be accreting hydrogen-poor material (ultracompact systems with white dwarf donors) never show evidence of hydrogen-rich bursts (Galloway et al. 2008). Some bursts from ultracompact systems are relatively long, but these "giant" (or "intermediate-duration") bursts exhibit dramatic photospheric radius expansion, thought to be produced by a thick layer of accumulated $\mathrm{He}$, which can accumulate only at low $\left(L_{X}<\right.$ $0.01 L_{\text {Edd }}$ ) accretion rates (in't Zand et al. 2005).

Globular clusters are highly efficient at producing X-ray binaries through dynamical interactions, such as the exchange of (heavy) NSs into pre-existing binary stars, replacing the lowermass star in the binary. Of perhaps 200 galactic LMXBs known to have reached $L_{X} \sim 10^{36} \mathrm{erg} \mathrm{s}^{-1}, 18$ (including Terzan $5 \mathrm{X}-3$ ) are located in globular clusters, a factor of $\sim 100$ overabundance per unit stellar mass compared to the galactic disk. LMXBs are concentrated in the densest, most massive globular clusters, which have the highest predicted rates of stellar interactions (e.g., Verbunt \& Hut 1987; Heinke et al. 2003c). Studying the number and types of LMXBs in different globular clusters can help us understand the dynamical processes that produce LMXBs in clusters. For example, identifying multiple LMXBs in one cluster has implications for interpreting observations of $\mathrm{X}$-ray emission from extragalactic globular clusters (such as their luminosity functions), where multiple LMXBs cannot be resolved (e.g., Sivakoff et al. 2007). Before Terzan 5 X-3, no more than two bright LMXBs had been identified in any one globular cluster (White \& Angelini 2001; Heinke et al. 2010; Pooley et al. 2010).

Terzan 5 is a dense and massive globular cluster close to the center of our Galaxy ( $d=5.9 \pm 0.5 \mathrm{kpc}$; Valenti et al. 2007), showing evidence of two separate stellar populations of different iron abundances, ages and helium content (Ferraro et al. 2009; D'Antona et al. 2010). Calculations of its stellar encounter rate suggest it may produce more X-ray binaries than any other Galactic globular cluster (Verbunt \& Hut 1987; Lanzoni et al. 2010; Bahramian et al. 2013). This status is supported by the largest population of known millisecond radio pulsars in any globular cluster, which are thought to be the descendants of LMXBs (Ransom et al. 2005; Hessels et al. 2006). Terzan 5 also hosts more than 50 known X-ray sources (Heinke et al. 2006b), including a dozen likely quiescent LMXBs (again the most numerous in any cluster).

Outburst of transient LMXBs have frequently been observed from Terzan 5 (Makishima et al. 1981; Warwick et al. 1988; Verbunt et al. 1995). Chandra observed one such outburst in 2000 (Heinke et al. 2003a), pinning down the location of an LMXB called EXO 1745-248, ${ }^{11}$ which was shown to have an unusually hard spectrum in quiescence during later Chandra observations (Wijnands et al. 2005; Degenaar \& Wijnands 2012). Another Terzan 5 outburst was identified in 2002 in

\footnotetext{
11 Note that the true identity of the transient seen by EXOSAT in the 1980s
} (leading to the EXO name) is not known.
RXTE All-Sky Monitor data (Wijnands et al. 2002a), but no imaging observations were taken. In 2010 an outburst was seen from an $11 \mathrm{~Hz}$ pulsar, IGR J17480-2446 (Terzan 5 X-2, Bordas et al. 2010; Strohmayer \& Markwardt 2010), leading to a variety of detailed studies of the orbit and spin period, bursts, spectrum, burst oscillations, and evolution (e.g., Papitto et al. 2011; Chakraborty et al. 2011; Miller et al. 2011; Motta et al. 2011; Cavecchi et al. 2011; Linares et al. 2011, 2012; Patruno et al. 2012; Papitto et al. 2012; Altamirano et al. 2012a). A Chandra observation identifying the outbursting source (Pooley et al. 2010) allowed follow-up observations to track the crustal cooling of the NS (Degenaar et al. 2011; Degenaar \& Wijnands 2011; Degenaar et al. 2013a), while the even more precise moon occultation position (Riggio et al. 2012) permitted identification of the near-IR counterpart (Testa et al. 2012). Another Terzan 5 outburst, in 2011, was identified as EXO 1745-248 through a Chandra observation (Pooley et al. 2011a), and showed a superburst (a very long and energetic X-ray burst, thought to be powered by the burning of a thick layer of carbon) at the beginning of the outburst (Serino et al. 2012; Altamirano et al. 2012b)

In this paper we identify and study the outburst of the third transient X-ray binary in the globular cluster Terzan 5, Swift J174805.3-244637 (henceforth Terzan 5 X-3). We detected this transient using Swift/XRT (Wijnands et al. 2012), and identified spectral hardening in the rise of the outburst (Heinke et al. 2012), a Type I X-ray burst (Altamirano et al. 2012c), and the quiescent X-ray counterpart in Chandra images (Homan \& Pooley 2012). In Section 2 we present the X-ray data used and describe our data extraction. In Section 3 we derive the position of Terzan 5 X-3 by comparing observations before and during the outburst, analyze the spectral variation of the persistent emission throughout the outburst, study the properties of the thermonuclear burst, and analyze its quiescent X-ray spectrum. Finally, we discuss our results in Section 4.

\section{DATA EXTRACTION}

\subsection{Swift/XRT}

We monitored Terzan 5 up to a few times per week for part of 2012 with the Swift/XRT, covering the $0.3-10 \mathrm{keV}$ energy range (Burrows et al. 2005), as part of our monitoring campaigns of globular cluster X-ray transients (see Altamirano et al. 2012b; D. Altamirano et al. 2013, in preparation). This monitoring enabled us to observe the rising outburst of a new transient (and the 3rd known transient LMXB in this cluster) first detected on 2012 July 6 (Wijnands et al. 2012).

We used Swift/XRT's photon counting (PC) mode, which produces two-dimensional images, and windowed timing (WT) mode for which CCD data is collapsed into a one-dimensional image for fast readout. PC mode data should be checked for pile-up when the count rate exceeds 0.5 count $\mathrm{s}^{-1}$. Pile-up is the recording of multiple photons as a single event, leading in the worst case to rejection of all events from the center of the pointspread function, or PSF. Our Swift/XRT observations include 22 observations during the outburst, with 8 observations in WT and the rest in PC mode (Table 1).

We used HEASOFT 6.12 and FTOOLS ${ }^{12}$ (Blackburn 1995) to reduce and analyze the data. We reprocessed the data with the FTOOLS xrtpipeline and manually extracted data for spectral analysis. We investigated every observation for pile-up,

\footnotetext{
12 http://heasarc.gsfc.nasa.gov/ftools/
} 
Table 1

List of Swift/XRT Observations (Top) and Chandra/ACIS Observations (Bottom) of Terzan 5 Used

\begin{tabular}{|c|c|c|c|c|c|}
\hline Obs. ID & Date & MJD & Exposure & $\begin{array}{l}\text { Avg. Count Rate } \\
\quad\left(\text { count s }{ }^{-1}\right)\end{array}$ & Notes \\
\hline \multicolumn{6}{|c|}{ Swift/XRT observations } \\
\hline 32148002 & 2012 Feb 9 & 55966.9 & $985 \mathrm{~s}$ & $1.75 \times 10^{-2}$ & PCmode; quiescent \\
\hline 91445001 & 2012 Jun 11 & 56089.8 & $913 \mathrm{~s}$ & $2.67 \times 10^{-2}$ & PCmode; quiescent \\
\hline 91445002 & 2012 Jun 16 & 56094.5 & $1028 \mathrm{~s}$ & $1.48 \times 10^{-2}$ & PCmode; quiescent \\
\hline 91445003 & 2012 Jun 21 & 56099.0 & $1033 \mathrm{~s}$ & $1.66 \times 10^{-2}$ & PCmode; quiescent \\
\hline 91445004 & 2012 Jun 26 & 56104.8 & $1050 \mathrm{~s}$ & $9.68 \times 10^{-3}$ & PCmode; quiescent \\
\hline 91445005 & 2012 Jun 30 & 56108.6 & $935 \mathrm{~s}$ & $1.40 \times 10^{-2}$ & PCmode; quiescent \\
\hline 91445006 & 2012 Jul 6 & 56114.8 & $1197 \mathrm{~s}$ & $7.65 \times 10^{-2}$ & PCmode; First detection of rise; (1) \\
\hline 32148003 & 2012 Jul 7 & 56115.8 & $987 \mathrm{~s}$ & 0.154 & PCmode; (2) \\
\hline 32148004 & 2012 Jul 8 & 56117.0 & $987 \mathrm{~s}$ & 0.245 & PCmode; (2) \\
\hline 32148005 & 2012 Jul 10 & 56118.1 & $781 \mathrm{~s}$ & 1.26 & PCmode; Piled up; (2) \\
\hline 32148006 & 2012 Jul 12 & 56120.7 & $978 \mathrm{~s}$ & 3.19 & PCmode; Piled up; Hard/soft transition \\
\hline 526511000 & 2012 Jul 13 & 56121.7 & $251 \mathrm{~s}$ & 11.4 & WTmode \\
\hline 91445008 & 2012 Jul 16 & 56124.3 & $253 \mathrm{~s}$ & 14.2 & WTmode \\
\hline 526892000 & 2012 Jul 16 & 56124.9 & $596 \mathrm{~s}$ & 14.9 & WTmode \\
\hline 32148007 & 2012 Jul 17 & 56125.9 & $960 \mathrm{~s}$ & $20.4(16.3)^{\mathrm{a}}$ & WTmode; Type I X-ray burst; (3) \\
\hline 91445009 & 2012 Jul 21 & 56129.1 & $1173 \mathrm{~s}$ & 71.8 & WTmode \\
\hline 91445010 & 2012 Jul 26 & 56134.1 & $612 \mathrm{~s}$ & 23.5 & PCmode; Piled up \\
\hline 91445011 & 2012 Aug 1 & 56140.1 & $985 \mathrm{~s}$ & 52.1 & WT mode \\
\hline 91445012 & 2012 Aug 5 & 56144.8 & $1057 \mathrm{~s}$ & 24.1 & WTmode; Return to hard state? \\
\hline 91445013 & 2012 Aug 10 & 56149.4 & $1031 \mathrm{~s}$ & 4.15 & WTmode \\
\hline 32148008 & 2012 Aug 11 & 56150.1 & $1488 \mathrm{~s}$ & 3.16 & PCmode; Piled up \\
\hline 32148011 & 2012 Aug 13 & 56152.2 & $1480 \mathrm{~s}$ & 2.47 & PCmode; Piled up \\
\hline 530808000 & 2012 Aug 13 & 56152.4 & $774 \mathrm{~s}$ & 2.97 & PCmode; Piled up \\
\hline 32148010 & 2012 Aug 14 & 56153.2 & $1494 \mathrm{~s}$ & 2.51 & PCmode; Piled up \\
\hline 91445014 & 2012 Aug 15 & 56154.3 & $1060 \mathrm{~s}$ & 2.17 & PCmode; Piled up \\
\hline 32148012 & 2012 Aug 19 & 56158.3 & $2153 \mathrm{~s}$ & 1.78 & PCmode; Piled up \\
\hline 91445015 & 2012 Aug 20 & 56159.1 & $1006 \mathrm{~s}$ & 2.14 & PCmode; Piled up \\
\hline 91445016 & 2012 Aug 24 & 56163.2 & $1131 \mathrm{~s}$ & 1.05 & PCmode \\
\hline 32148013 & 2012 Aug 30 & 56169.3 & $2075 \mathrm{~s}$ & $2.95 \times 10^{-2}$ & PCmode; quiescent \\
\hline 32148014 & 2012 Sep 13 & 56177.6 & $1953 \mathrm{~s}$ & $2.02 \times 10^{-2}$ & PCmode; quiescent \\
\hline 32148015 & 2012 Sep 20 & 56184.6 & $1976 \mathrm{~s}$ & $1.98 \times 10^{-2}$ & PCmode; quiescent \\
\hline \multicolumn{6}{|c|}{ Chandra/ACIS observations } \\
\hline 3798 & 2003 Jul 13 & 52833.6 & $39.34 \mathrm{ks}$ & $9.91 \times 10^{-3}$ & quiescent; $(4,5)$ \\
\hline 10059 & 2009 Jul 15 & 55027.7 & $36.26 \mathrm{ks}$ & $7.42 \times 10^{-3}$ & quiescent; $(6,7)$ \\
\hline 13225 & 2011 Feb 17 & 55609.3 & $29.67 \mathrm{ks}$ & $5.56 \times 10^{-3}$ & quiescent; $(6,7)$ \\
\hline 13252 & 2011 Apr 29 & 55680.7 & $39.54 \mathrm{ks}$ & $6.78 \times 10^{-3}$ & quiescent; $(7,8)$ \\
\hline 13705 & 2011 Sep 5 & 55809.7 & $13.87 \mathrm{ks}$ & $5.69 \times 10^{-3}$ & quiescent; (9) \\
\hline 14339 & 2011 Sep 8 & 55812.1 & $34.06 \mathrm{ks}$ & $6.08 \times 10^{-3}$ & quiescent; (9) \\
\hline 13706 & 2012 May 13 & 56060.7 & $46.46 \mathrm{ks}$ & $7.58 \times 10^{-3}$ & quiescent; (9) \\
\hline 13708 & 2012 Jul 30 & 56138.4 & $9.84 \mathrm{ks}$ & 6.60 & Terzan 5 X-3 outburst; Piled up; $(10,11)$ \\
\hline
\end{tabular}

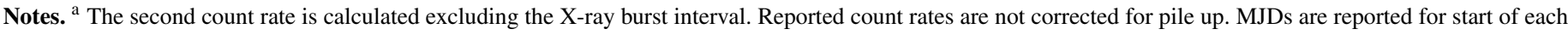
observation.

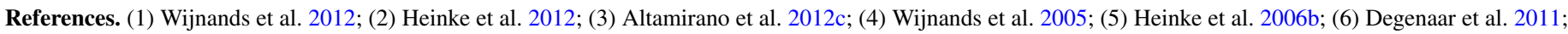
(7) Degenaar \& Wijnands 2011; (8) Degenaar \& Wijnands 2012; (9) Degenaar et al. 2013a; (10) Homan \& Pooley 2012; (11) this work.

following the Swift/XRT pile-up thread, ${ }^{13}$ and extracted data from an annulus around the source in PC mode observations that suffered from pile-up. We subtracted background from a circular region in the vicinity of the source in all PC observations. The extraction region for WT data was chosen to be a box around the event array (background subtraction was unnecessary for these count rates), as discussed in the Swift/XRT data reduction guide. ${ }^{14}$ We extracted spectra in the $0.5-10 \mathrm{keV}$ bandpass using FTOOLS xselect, and created ancillary response function (ARF) files for each observations using FTOOLS xrtmkarf. We performed spectral analysis using XSPEC 12.7.1 (Arnaud 1996).

\footnotetext{
$\overline{13}$ http://www.swift.ac.uk/analysis/xrt/pileup.php

$14 \mathrm{http}: / /$ heasarc.nasa.gov/docs/swift/analysis
}

For heavily absorbed sources, WT data show low energy spectral residuals, which look like a "bump" in the spectrum, and cause spectral uncertainties in the $\lesssim 1.0 \mathrm{keV}$ region. ${ }^{15}$ We compared Swift/XRT WT mode data to Chandra data (Section 2.2) taken within a few days, fitting them with the same model to find the energy range in which discrepancies appear. Based on this comparison, we ignored data below $1.4 \mathrm{keV}$ in all WT observations during the outburst.

\subsection{Chandra/ACIS}

We observed Terzan 5 X-3 during outburst with Chandra/ ACIS in full-frame and FAINT telemetry mode with no grating (Obs. ID: 13708, PI: Pooley). We also used Chandra archival

\footnotetext{
15 http://www.swift.ac.uk/analysis/xrt/digest_cal.php
} 

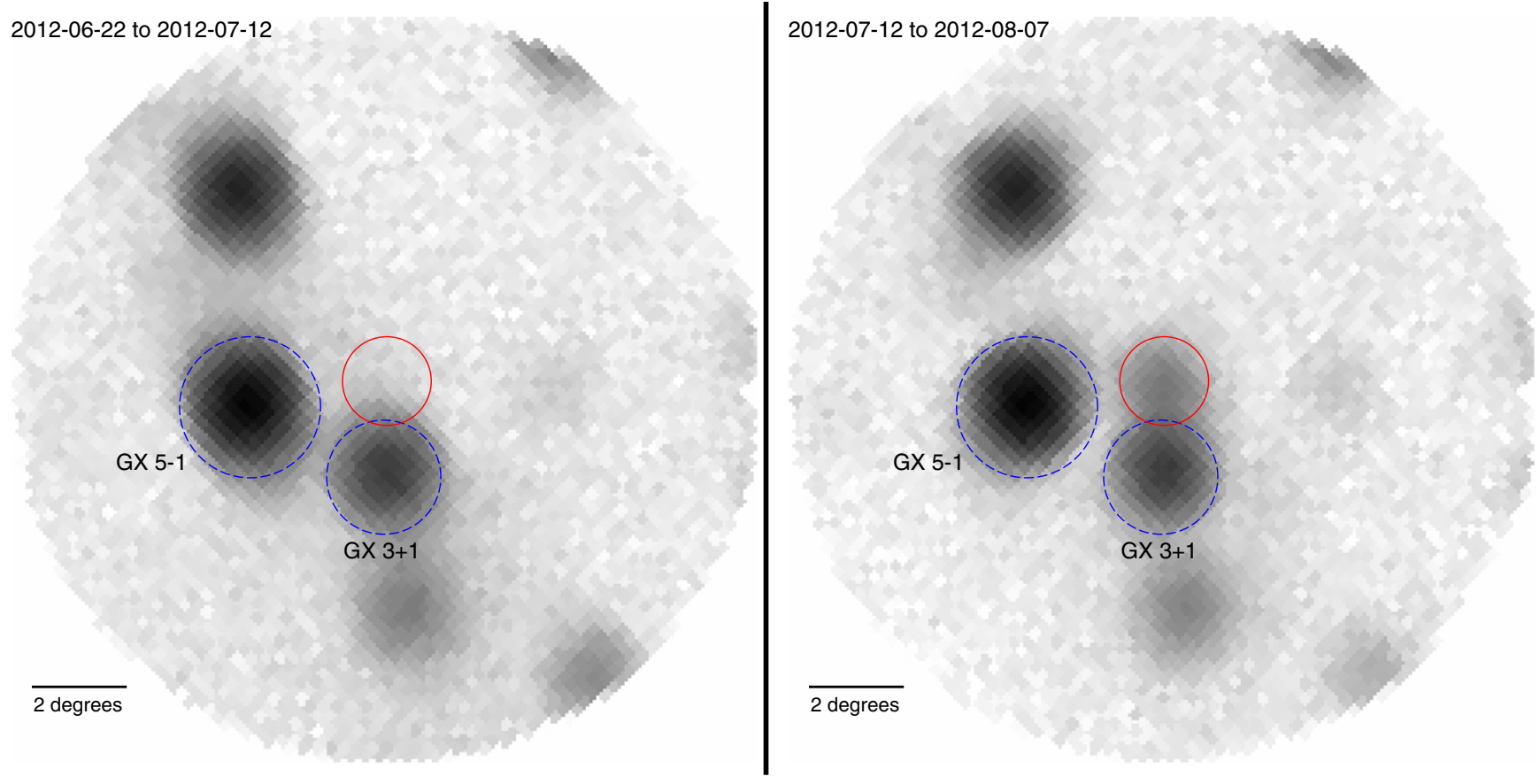

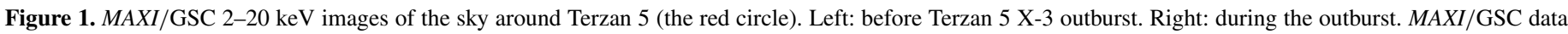
for Terzan 5 may suffer contamination from the nearby sources GX 5-1 and GX 3+1 (blue dashed circles).

(A color version of this figure is available in the online journal.)

data for our analysis of this source, details of which can be found in Table 1. All archival observations were taken in FAINT telemetry mode with the ACIS-S3 CCD at the focus. We analyze Chandra/ACIS data in the $0.5-10 \mathrm{keV}$ energy range.

Data was reprocessed using CIAO 4.4 (Fruscione et al. 2006), with CALDB 4.4.8, following the standard CIAO science threads. ${ }^{16}$ We used observations during which all sources in the globular cluster were in quiescence. We reprocessed the data, corrected the relative astrometry and ran CIAO reproject_events, and then stacked the event files together using CIAO dmmerge.

Spectra were then extracted from both the archival and new data using CIAO task dmextract. Terzan 5 X-3 was heavily piled up in the new observation (in outburst), and so we extracted a spectrum from the readout streak. Finally, we combined all archival (quiescent data) using FTOOLS addspec. ${ }^{17}$ Combining the quiescent data resulted in $240 \mathrm{ks}$ of exposure time.

\section{3. $M A X I / G S C$}

The MAXI all sky X-ray monitor's (Matsuoka et al. 2009) GSC detector data covers the $2-20 \mathrm{keV}$ energy range, and oneday averaged light curves are publicly provided in four bands: 2-4 keV, 4-10 keV, 10-20 keV and 2-20 keV (Mihara et al. 2011). We noticed two problems with $M A X I / G S C$ light curves. Due to the low spatial resolution of $M A X I / G S C$ and bright sources in the crowded field of Terzan 5, there is the possibility of background contamination from nearby sources like GX 3+1 and GX 5-1 (Figure 1). Since these two sources showed stable $\mathrm{X}$-ray brightness with no obvious variations in the $M A X I / G S C$ data during Terzan 5 X-3 outburst, the background contamination may lead to a constant enhanced background.

We also noticed that $M A X I / G S C$ light curves show periodic behavior with a period of $\approx 35$ days for various well-known

\footnotetext{
16 http://cxc.harvard.edu/ciao/threads/index.html

17 We found the results from CIAO's combine_spectra and FTOOLS's addspec completely identical.
}

stable X-ray sources (e.g., the Crab nebula). This is caused by calibration issues regarding the 70-day precession of the International Space Station's orbit (MAXI team 2013, private communication). This problem principally affects the $2-4 \mathrm{keV}$ data, with less effect on the 4-10 and 10-20 keV light curves. Thus we ignored the 2-4 keV light curves for this research. We also ignored MAXI/GSC 10-20 keV band light curves, due to the low statistical significance of Terzan 5 X-3's detection there. We decontaminated the MAXI/GSC 4-10 keV light curve assuming a constant background count rate of 0.023 , calculated based on a weighted average of the count rates before the outburst for a period of $\sim 120$ days, with the addition of a systematic error based on the rms variations in the light curve before the outburst. We used the corrected values of the statistical uncertainties in $M A X I / G S C$ data, as the MAXI team announced an erratum in the reported statistical uncertainties on 2013 April 26 (MAXI team 2013, private communication) noting that the corrected uncertainties are a factor of two larger than previously reported.

\subsection{Swift/BAT}

The Swift/BAT telescope data covers 15-150 keV energy range (Barthelmy et al. 2005). We used daily light curves from the Swift/BAT transient monitor results provided by the Swift/ BAT team (Krimm et al. 2013). Data points on these daily light curves are from Swift/BAT survey data and are represented in a single band (15-50 keV). These points are the weighted average of all observations performed each day. The Swift/ BAT has better angular resolution (20 vs.1 degree FWHM for Swift/BAT versus MAXI/GSC). Such an improvement in the angular resolution limits the chances of contamination occurring. Although we cannot rule out some contamination, it seems reasonable that it does not pose a serious problem. As such, it is not surprising that we do not see evidence of contamination of the Swift/BAT data by nearby sources as is identifiable in the MAXI data. 


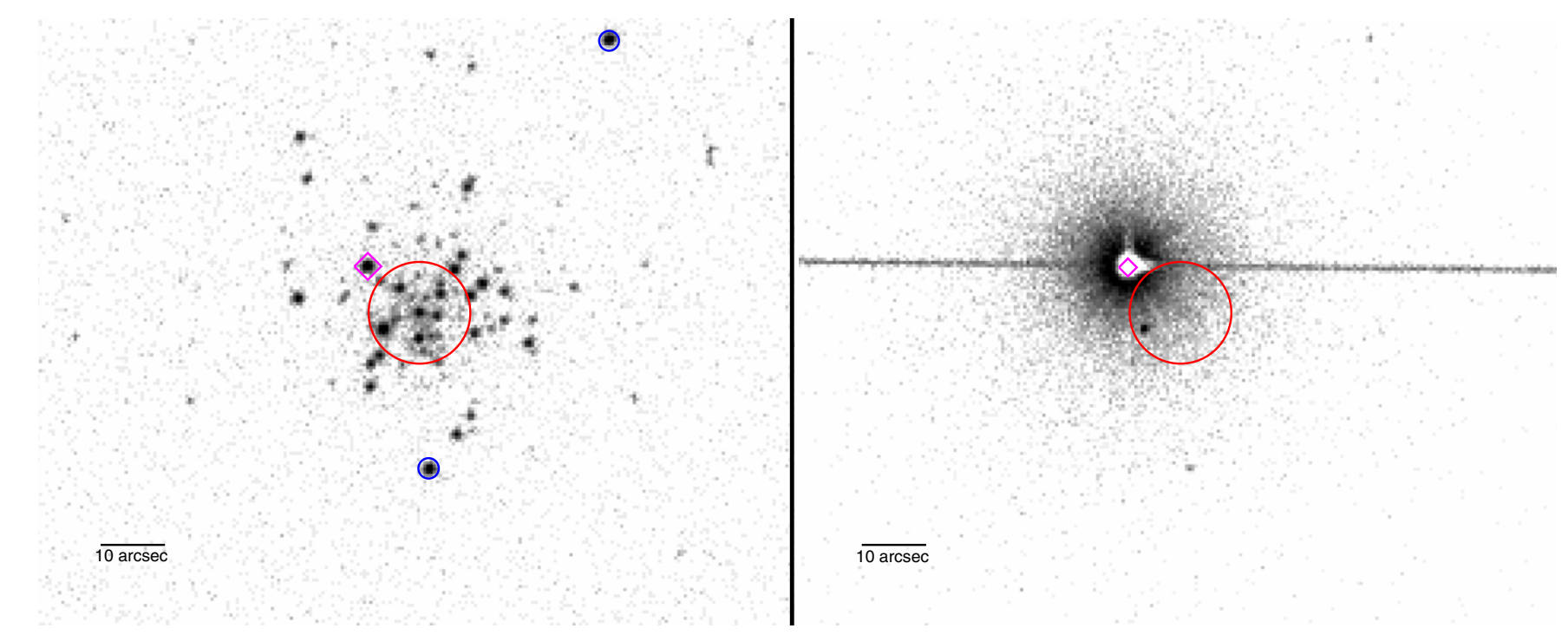

Figure 2. Chandra/ACIS observations of Terzan 5. Left: stacked image of observations during quiescence (bottom of Table 1) of all sources; total exposure $\approx 240 \mathrm{ks}$. Right: outburst of Terzan 5 X-3 (Obs. ID 13708 with exposure $\approx 10 \mathrm{ks}$ ). The red circles and magenta diamond represent the core of Terzan 5 (Harris 1996 ) and the position of Terzan 5 X-3, respectively (before astrometric corrections to the outburst image). Blue circles identify additional sources (CXOGLB J174804.7-244709 and CXOGLB J174802.6-244602) used for constraining the hydrogen column density of Terzan 5 (Section 3.3.1).

(A color version of this figure is available in the online journal.)

\section{ANALYSIS AND RESULTS}

\subsection{Position}

We accurately and precisely located the position of Terzan 5 $\mathrm{X}-3$, using the Chandra/ACIS data. We compared the Chandra/ ACIS observation taken during the outburst and a stacked image of 7 Chandra/ACIS observations taken when all Terzan 5 sources were in quiescence (Section 2.2, Table 1). We corrected the astrometry in the outburst observation by comparing the positions (using the CIAO wavdetect tool) of three other sources in this observation with their astrometrically corrected positions as reported in Heinke et al. (2006b). Using a weighted average of the required shifts (+0!'23 for R.A. and +0' 04 for Decl.), we find the position of Terzan 5 X-3 to be R.A. $=17: 48: 05.41 \pm 0.02$ and Decl. $=-24: 46: 38^{\prime \prime} .0 \pm 0.2$, in agreement $(2 \sigma)$ with the published position of the X-ray source CXOGLB J174805.4244637 (Heinke et al. 2006b), at R.A. $=17: 48: 05.413 \pm 0.001$ and Decl. $=-24: 46: 37.67 \pm 0$ s.02 (Figure 2).

\subsection{Phases of the Outburst}

We used MAXI/GSC and Swift/BAT hard X-ray transient monitor light curves to study the evolution of Terzan $5 \mathrm{X}-3$ 's outburst in the soft X-ray (4-10 keV, MAXI/GSC) and hard $\mathrm{X}$-ray $(15-50 \mathrm{keV}$, Swift/BAT) bands. We converted count rates into equivalent fluxes from the Crab Nebula to make these light curves suitable for comparison. For this purpose, we used conversion coefficients given for each instrument ${ }^{18}$ : for the Swift/BAT hard X-ray transient monitor $1 \mathrm{Crab}=$ 0.22 count $\mathrm{cm}^{-2} \mathrm{~s}^{-1}$ and for the MAXI/GSC 4-10 keV band 1 $\mathrm{Crab}=1.24$ count $\mathrm{cm}^{-2} \mathrm{~s}^{-1}$. We plot the light curves for Terzan 5 X-3's outburst as seen by both MAXI/GSC and Swift/BAT, and their ratio, in Figure 3.

We distinguish four phases of Terzan 5 X-3's outburst. (a) Rise: the hard X-ray brightness of the source increases, the source eventually becoming significantly detected in the soft

\footnotetext{
18 MAXI- http://maxi.riken.jp/top/index.php?cid=000000000036 Swift/BAThttp://swift.gsfc.nasa.gov/docs/swift/results/transients
}

X-ray as well. (b) Hard state: hard X-ray brightness reaches its peak. (c) Soft state: soft X-ray brightness peaks while hard $\mathrm{X}$-ray brightness drops. (d) Decline: the source briefly gets brighter in the hard X-ray again before turning off. Unfortunately there is insufficient data from the MAXI /GSC during the decline of the outburst. Therefore, we are unable to confirm that Terzan $5 \mathrm{X}-3$ returns to the hard state during its decline.

We used the soft X-ray (MAXI/GSC 4-10 keV, $S$ ) and hard X-ray (Swift/BAT 15-50 keV, H) light curves, to create a color-luminosity diagram (as an analogy to a hardness-intensity diagram, Fender et al. 2004) for the outburst (Figure 4). We defined our color to be $(H-S) /(H+S)$, and defined luminosity as $(H+S)$, converting count rates to luminosities in each band before their summation or subtraction. We converted count rates to luminosities with the assumption of a $5.9 \mathrm{kpc}$ distance and power-law spectra, using power-law index values inferred from Swift/XRT data spectral fitting (Section 3.3.2). We extrapolated the MAXI/GSC 4-10 keV band flux to $0.1-12 \mathrm{keV}$ and the Swift/BAT $15-50 \mathrm{keV}$ band flux to $12-50 \mathrm{keV}$, and calculated luminosities in the $0.1-50 \mathrm{keV}$ band. We cannot measure the spectral index above $10 \mathrm{keV}$, but since in the hard state the spectra are typically reasonably described by a power-law up to $50 \mathrm{keV}$, and in the soft state the flux above $12 \mathrm{keV}$ is a minor contribution to the total, this is unlikely to have a large effect. In the $0.5-10 \mathrm{keV}$ band, the source was bright for approximately 20 days, reaching a maximum luminosity of $7 \times 10^{37} \mathrm{erg} \mathrm{s}^{-1}$ and an average luminosity of $3 \times 10^{37} \mathrm{erg} \mathrm{s}^{-1}$ in this time interval. The evolution of the outburst and phases mentioned above can be clearly seen in this color-luminosity diagram (Figure 4). Spectral evolution during the outburst, including at fainter fluxes but with a more limited bandpass, can also be seen in Swift/XRT observations (Section 3.3.2).

\subsection{Spectral Analysis 3.3.1. Hydrogen Column Density of Terzan 5}

The first step in our spectral analysis is constraining hydrogen column density $N_{\mathrm{H}}$, which we check by spectral analysis of multiple sources in the cluster. Since the sources are located 


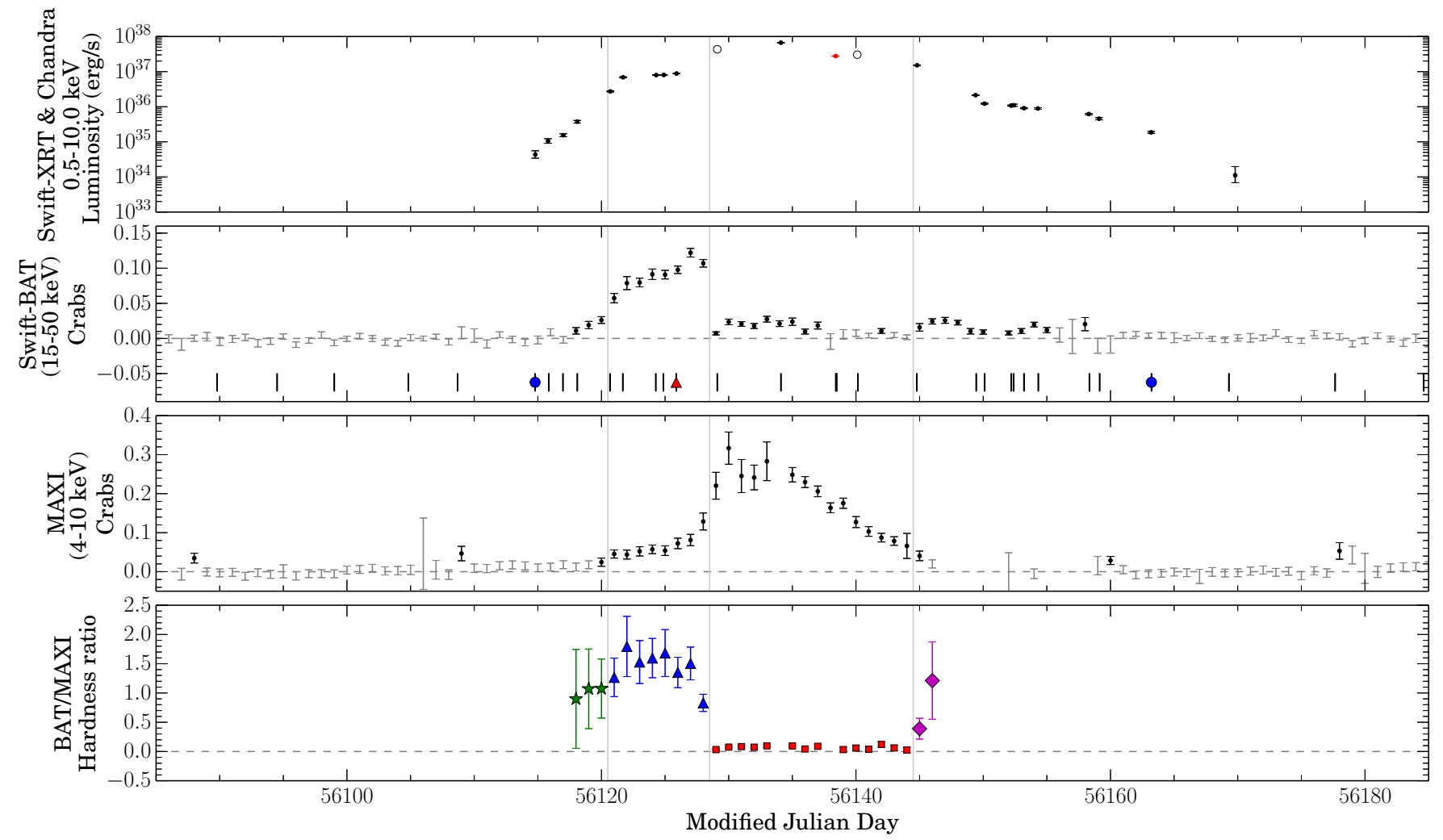

Figure 3. Evolution of Terzan 5 X-3 outburst. From the top, first panel: luminosities from spectral fitting of Swift/XRT and Chandra/ACIS pointed observations (Section 3.3.2). Empty circles represent observations where these fits were poor $\left(\chi_{v}^{2}>2\right)$. The red data point represents Chandra/ACIS observation. Second panel: Swift/BAT light curve in the 15-50 keV band. Third panel: MAXI/GSC background-subtracted light curve in the 4-10 keV band. Fourth panel: hardness ratio (H/S after conversion to Crab units) of the two light curves (Swift/BAT/MAXI/GSC). Black data points in the upper two panels represent significant detections ( $>2 \sigma$ ) in each band, while gray bars show times when Terzan 5 X-3 was not significantly detected. Vertical lines at the bottom of the first panel represent times of pointed Swift/XRT and Chandra/ACIS observations. The first and last detections of the outburst in Swift/XRT data are represented by blue circles. The red triangle identifies the time of the detected thermonuclear burst (Section 3.3.3). Colors and shapes in the bottom panel indicate the different phases of outburst: (a) green stars, rise, (b) blue triangles, hard state, (c) red squares, soft state, (d) magenta diamonds, decline. Both Swift/BAT and MAXI/GSC light curves are in Crab units, and error bars are $1 \sigma$ uncertainties. Vertical lines in panels show approximate boundary of introduced phases. Note that in the fourth panel symbol size is larger than the error bars in the soft state. All daily averages are plotted at the beginning of each day.

(A color version of this figure is available in the online journal.)

within $1^{\prime}$ of each other, we expect little variation in $N_{\mathrm{H}}$ along the different sightlines. Except for LMXBs observed at high inclination, generally the measured $N_{\mathrm{H}}$ throughout an outburst appears to be stable (Miller et al. 2009), so we assume that Terzan 5 X-3's $N_{\mathrm{H}}$ remains constant. We used Chandra/ACIS observations of Terzan 5 taken when all sources were quiescent (Table 1). We extracted spectra of three of the brighter faint sources in Terzan 5 (Terzan 5 X-3, CXOGLB J174804.7244709 and CXOGLB J174802.6-244602; Heinke et al. 2006b; Figure 2) from each observation using CIAO dmextract and combined the extracted spectra for each source. We fit these combined spectra, along with the Chandra spectrum of Terzan 5 X-3 during the outburst, simultaneously. We used appropriate models based on previous studies of each faint source (Heinke et al. 2006b), confirmed as acceptable fits. For Terzan 5 X-3 during quiescence, and for CXOGLB J 174804.7-244709, we used a NS atmosphere (NSATMOS; Heinke et al. 2006a) plus a power-law (PEGPWRLW). For CXOGLB J 174802.6-244602 we used a power-law. Finally, for Terzan 5 X-3 during outburst, we used a disk model (DISKBB) plus a thermal Comptonization model (COMPTT; Titarchuk 1994). We fit all these spectra simultaneously with a single value of $N_{\mathrm{H}}$, using the PHABS model in XSPEC, with Anders \& Grevesse (1989) abundances, finding $N_{\mathrm{H}}=1.74_{-0.08}^{+0.06} \times 10^{22} \mathrm{~cm}^{-2}$. Individual spectral fits gave consistent results, with differences below the $10 \%$ level. This value is consistent with the measurements in Heinke et al. (2003a), Wijnands et al. (2005), Heinke et al. (2006b), and Degenaar et al. (2011), though not with the lower value of Miller et al. (2011), ${ }^{19}$ and is consistent with the $E(B-V)$ estimates in Valenti et al. (2007) and Massari et al. (2012), using the Güver \& Özel (2009) relation to $N_{\mathrm{H}}$. We note that Miller et al. (2011) fit a simple blackbody plus power-law to the outburst spectrum of IGR J17480-2446 (Terzan 5-X2), which may lead to systematic differences compared to more complex outburst spectral models. We used our best-fit $N_{\mathrm{H}}$ value in the PHABS model, using default abundances, for the rest of the spectral analysis throughout this paper.

For comparison, we also used the same procedure for constraining $N_{\mathrm{H}}$ using the TBABS model (instead of PHABS), with Wilms et al. (2000) abundances, finding an increase in $N_{\mathrm{H}}$ to $2.6 \pm 0.1 \times 10^{22} \mathrm{~cm}^{-2}$. The remaining parameters agreed within the errors with the results using our default absorption model, indicating that for our level of analysis only internal consistency is required in the choice of absorption model.

\subsubsection{Outburst}

The spatial resolution of Swift/XRT is such that we must account for possible contamination due to emission form other $\mathrm{XRBs}$ in the cluster. To assess these levels, we fit the spectra

19 Miller et al. (2011) report $N_{\mathrm{H}}=(1.17 \pm 0.04) \times 10^{22} \mathrm{~cm}^{-2}$, though they do not report the abundance scale they use. 


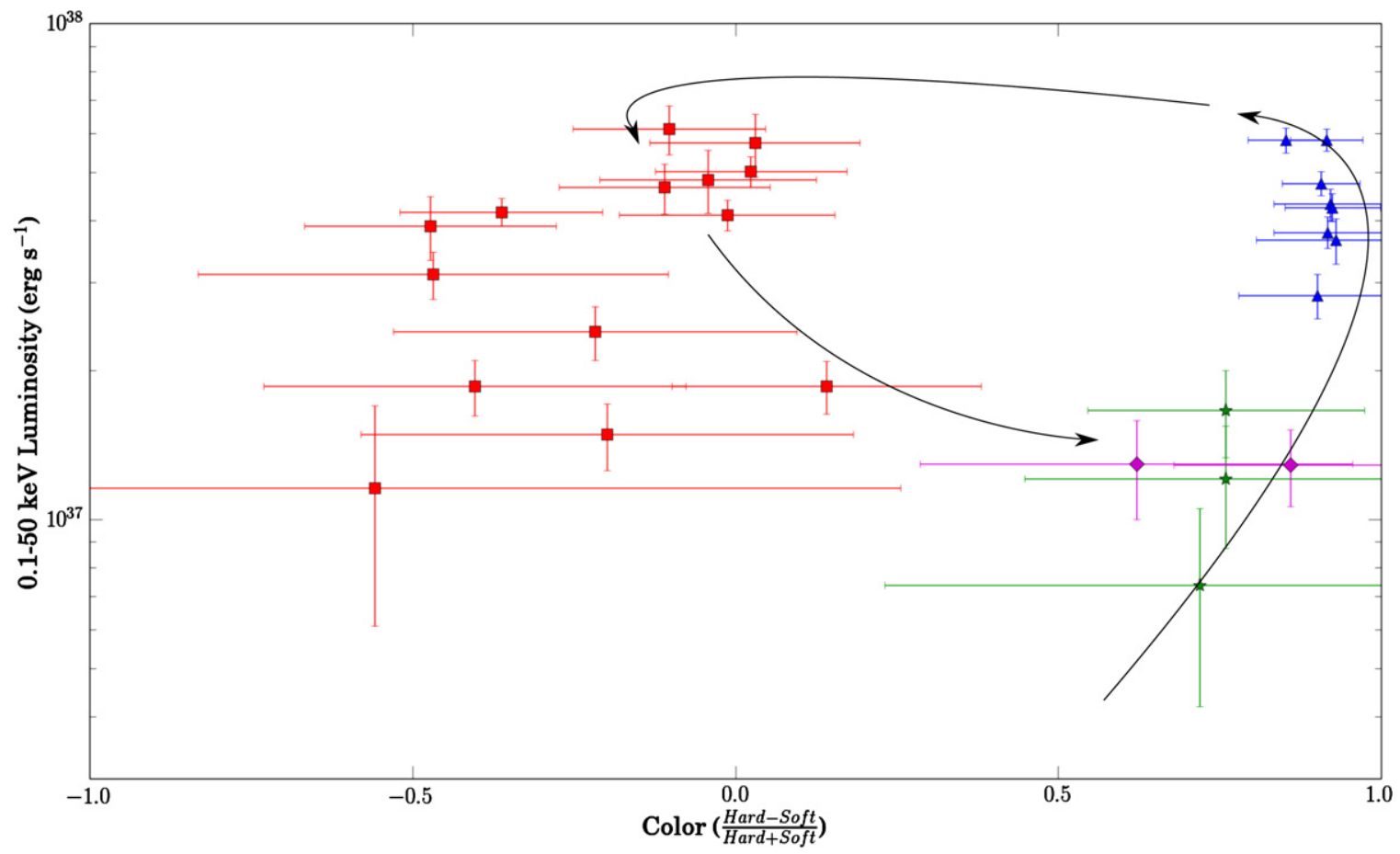

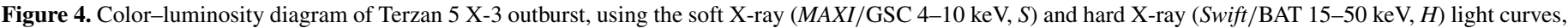

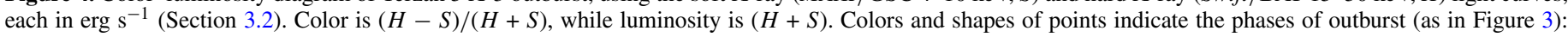

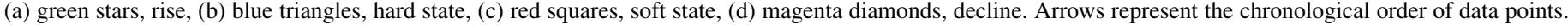
Error bars indicate $1 \sigma$ uncertainties.

(A color version of this figure is available in the online journal.)

of pre-outburst observations (using the same extraction region as for the outburst spectra), finding a background level of $L_{X} \sim 10^{34} \mathrm{erg} \mathrm{s}^{-1}$. We used 6 Swift/XRT observations taken before the outburst to estimate the combined spectrum from the other cluster sources. Due to the low number of counts per observation, we combined their spectra. We fit the resulting preoutburst spectrum with an absorbed power-law model (Table 2) with $N_{\mathrm{H}}$ fixed to $1.74 \times 10^{22} \mathrm{~cm}^{-2}$, based on the results in Section 3.3.1.

For our initial spectral analysis, we used an absorbed powerlaw model to fit Terzan 5 X-3's spectrum, including a second power-law component with values fixed to the pre-outburst results to model the background (Table 2, Figure 5). A simple power-law model provided a good fit to most of the spectra (Table 2), while physically motivated complex spectral models could not be well-constrained for the high-quality bright outburst spectra (see below), so we focus on the results from power-law fits. This spectral analysis shows a significant drop of photon index from $2.6 \pm 0.7$ to $1.4 \pm 0.1$ during the outburst rise (Figure 5), showing a clear hardening of the spectrum during the rise from quiescence. During the hard state, the photon index shows no significant variations and is $\approx 1.4$. After the phase transition to the soft state, the photon index softens to $\approx 1.9$, with significant variations and several poor fits.

Several spectral models have been suggested in the literature to model the detailed spectra of transient NS LMXBs in outburst, see e.g., Lin et al. (2007). These models usually contain a soft component for the radiation from the disk and/or boundary layer(i.e., a multi-color black body) and a hard component for the radiation from the hot corona around the accreting object (i.e., Comptonized radiation). We attempted to perform analyses of Terzan 5 X-3's outburst spectra using a variety of complex models with multiple components (e.g., DISKBB + COMPTT,
BBODY+COMPTT, BBODY+BKNPOW). We could not obtain strong constraints on the spectral parameters due to the limited energy band available and limited statistical quality of the XRT data. The Swift/XRT WT spectra suffered particularly from calibration issues below $\sim 1 \mathrm{keV}$ (Section 2.1). The Swift/ BAT survey mode hard $(>15 \mathrm{keV})$ X-ray spectra had a low signal to noise ratio, which prohibited spectral analysis. We defer further detailed spectral fitting, e.g., to clearly distinguish thermal versus nonthermal components, to future work.

\subsubsection{Thermonuclear Burst}

During Swift/XRT ObsID 32148007, which started at 20:54:00 UT on 2012 July 17, we detected an eightfold count rate increase over $\sim 3 \mathrm{~s}$, starting at $\approx 21: 06: 40$, followed by a slower decline over $\sim 1$ minute (Figure 6 , bottom panel), suggestive of a thermonuclear burst (Altamirano et al. 2012c). Using FTOOLS $x$ select, we divided the data from this observation into time intervals of $4 \mathrm{~s}$ each. We extracted spectra from each time interval using FTOOLS xselect and analyzed the spectra.

During this thermonuclear burst, the count rate reached $\approx 160$ count $\mathrm{s}^{-1}$. There is a possibility of pileup in Swift/XRT observations in windowed timing mode when the count rate exceeds 100 count s ${ }^{-1}$ (Romano et al. 2006). Following the Swift/XRT pile-up thread for WT data, we extracted spectra while excluding increasingly large fractions of the central PSF. Doing this, we found that the fitted photon index did not change, and thus we found no significant signs of pileup in this observation during the burst.

We fit an absorbed power-law to the spectrum extracted from a pre-burst interval, and considered this fit as a fixed component of the spectral model for time intervals during the burst (cf., Worpel et al. 2013; our statistics are insufficient to determine whether this assumption is correct, and moderate changes should 


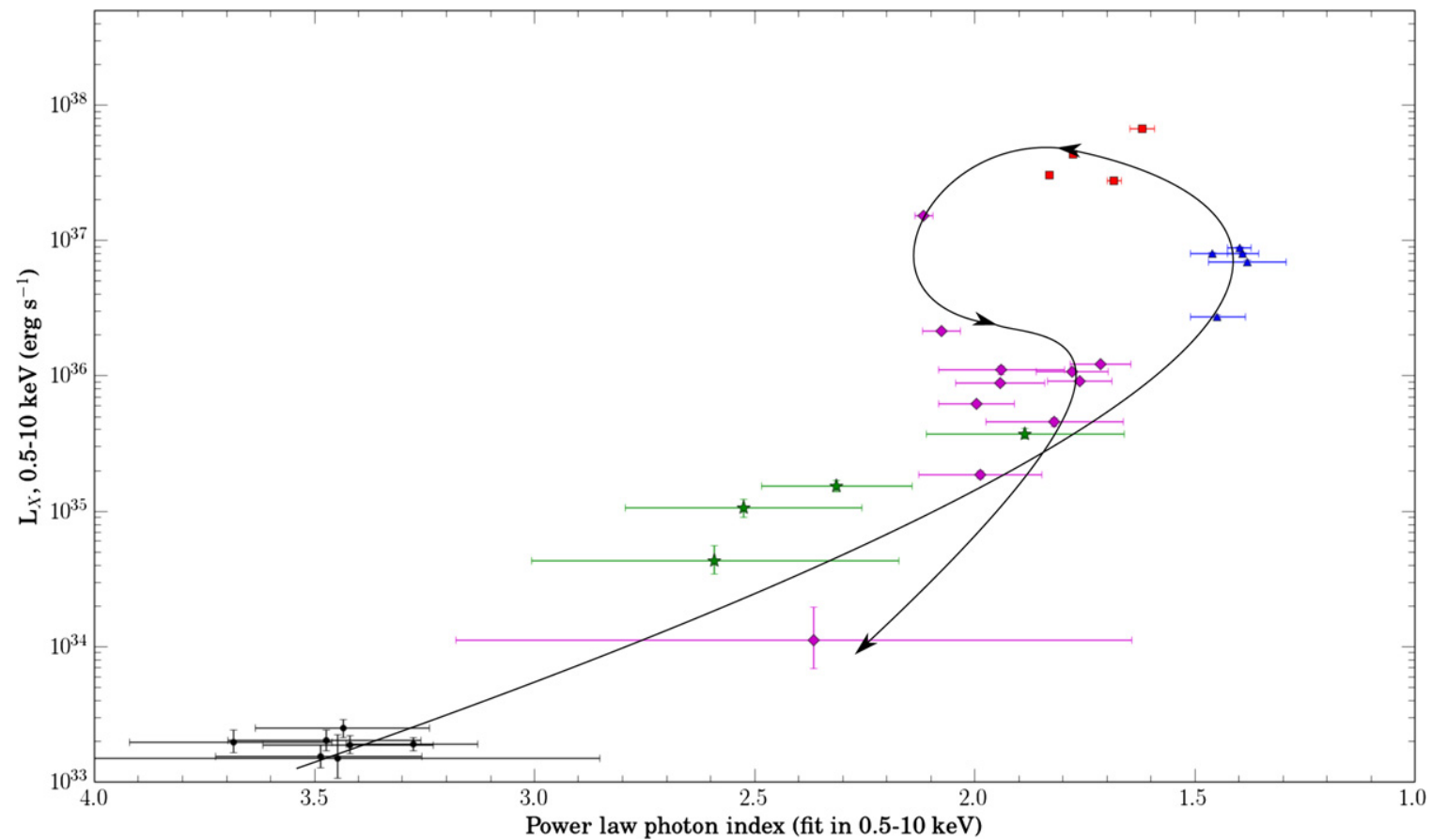

Figure 5. Luminosity vs. power-law photon index of Terzan 5 X-3 during the outburst, using Swift/XRT and one Chandra/ACIS outburst observations plus two quiescent Chandra/ACIS observations. Colors and shapes of points indicate the phases of outburst (as in Figure 3): (a) green stars, rise, (b) blue triangles, hard state, (c) red squares, soft state, (d) magenta diamonds, return to hard state. The black circles represent Chandra/ACIS observations in quiescence, covering the range of observed hardness in the quiescent state. Arrows represent the chronological order of data points. Error bars indicate $1 \sigma$ uncertainties.

(A color version of this figure is available in the online journal.)

Table 2

Swift/XRT and Chandra/ACIS Observations Fit to an Absorbed Power-Law Model

\begin{tabular}{|c|c|c|c|c|c|}
\hline Obs. ID & MJD & Photon Index & Flux & $L_{X}$ & $\chi_{v}^{2} /$ D.O.F.(nhp) \\
\hline $32148002-91445005$ & 55966-56108 & $2.4_{-0.4}^{+0.5}$ & $2.4_{-0.6}^{+0.7}$ & $1.0_{-0.2}^{+0.3}$ & $0.68 / 7(0.69)$ \\
\hline 91445006 & 56114.8 & $2.6 \pm 0.7$ & $10_{-3}^{+5}$ & $4_{-1}^{+2}$ & $0.83 / 6(0.54)$ \\
\hline 32148004 & 56117.0 & $2.3 \pm 0.3$ & $37 \pm 6$ & $15 \pm 2$ & $1.86 / 9(0.052)$ \\
\hline 32148005 & 56118.1 & $1.9 \pm 0.4$ & $90 \pm 12$ & $37 \pm 5$ & $0.54 / 6(0.78)$ \\
\hline 32148006 & 56120.7 & $1.4 \pm 0.1$ & $658 \pm 32$ & $273 \pm 13$ & $1.58 / 19(0.05)$ \\
\hline 526892000 & 56124.9 & $1.39 \pm 0.06$ & $1936 \pm 46$ & $803 \pm 19$ & $1.56 / 34(0.03)$ \\
\hline 32148007 & 56125.9 & $1.40 \pm 0.04$ & $2125 \pm 40$ & $882 \pm 17$ & $1.05 / 37(0.38)$ \\
\hline $91445009^{\mathrm{a}}$ & 56129.1 & $1.8 \pm ?$ & $10440 \pm ?$ & $4333 \pm ?$ & $3.94 / 56\left(1.9 \times 10^{-21}\right)$ \\
\hline 91445010 & 56134.1 & $1.62 \pm 0.05$ & $16070 \pm 330$ & $6669 \pm 137$ & $0.77 / 36(0.84)$ \\
\hline $13708^{\mathrm{a}}(\mathrm{CXO})$ & 56138.4 & $1.68 \pm 0.03$ & $6680 \pm 80$ & $2772 \pm 33$ & $1.76 / 102\left(3.4 \times 10^{-6}\right)$ \\
\hline $91445011^{\mathrm{a}}$ & 56140.1 & $1.8 \pm ?$ & $7330 \pm ?$ & $3042 \pm ?$ & $2.10 / 80\left(3.4 \times 10^{-6}\right)$ \\
\hline 91445012 & 56144.8 & $2.11 \pm 0.03$ & $3651 \pm 70$ & $1515 \pm 29$ & $1.12 / 38(0.27)$ \\
\hline $32148010^{\mathrm{a}}$ & 56153.2 & $1.8 \pm 0.1$ & $219 \pm 13$ & $91 \pm 5$ & $1.79 / 44\left(9.5 \times 10^{-4}\right)$ \\
\hline 91445014 & 56154.3 & $1.9 \pm 0.2$ & $215 \pm 18$ & $89 \pm 7$ & $1.06 / 23(0.38)$ \\
\hline 32148012 & 56158.3 & $2.0 \pm 0.1$ & $149 \pm 11$ & $62 \pm 5$ & $1.06 / 33(0.37)$ \\
\hline 91445015 & 56159.1 & $1.8 \pm 0.3$ & $110 \pm 13$ & $46 \pm 5$ & $0.97 / 10(0.47)$ \\
\hline 91445016 & 56163.2 & $2.0 \pm 0.2$ & $45 \pm 5$ & $19 \pm 2$ & $1.49 / 16(0.09)$ \\
\hline 32148013 & 56169.3 & $2.4_{-1.2}^{+1.5}$ & $2.7_{-1.5}^{+4.8}$ & $1.1_{-0.6}^{+1.9}$ & $\ldots$ \\
\hline
\end{tabular}

Notes. Unabsorbed flux in units of $10^{-12} \mathrm{erg} \mathrm{s}^{-1} \mathrm{~cm}^{-2}$ and luminosity in $10^{34} \mathrm{erg} \mathrm{s}^{-1}$, both in $0.5-10 \mathrm{keV}$. All the flux and luminosity values are background subtracted. "a" indicates spectra which are not well described by a power law (null hypothesis probability is $<10^{-2}$ ); if $\chi^{2}>2$, no errors are calculated (indicated by “?”). The first row shows a spectral fit to a merged spectrum of 6 Swift/XRT observations of Terzan 5 before the outburst of Terzan 5 X-3. For Obs.ID 32148013, due to low counts, we used cstat statistics in the fitting. Reported uncertainties are $90 \%$ intervals. 


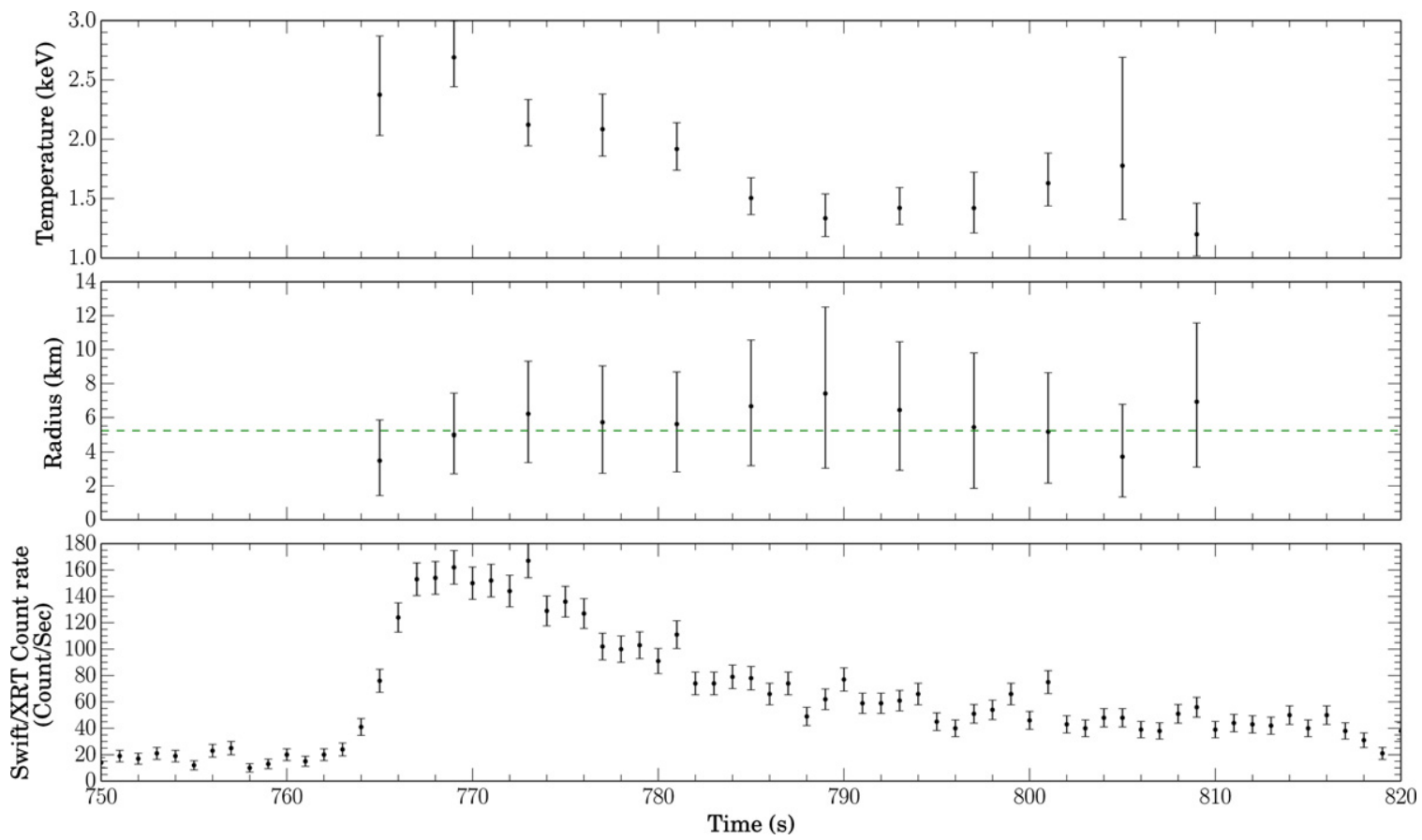

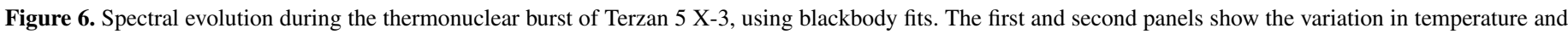

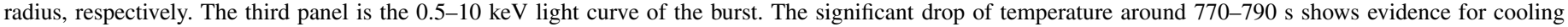

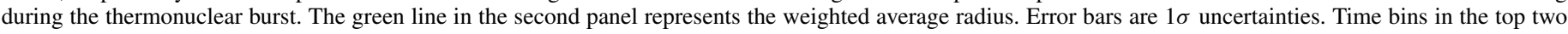
panels are $4 \mathrm{~s}$ long.

(A color version of this figure is available in the online journal.)

not affect our conclusions). We fit an absorbed blackbody model (BBODYRAD) to the burst emission, finding decent fits for all intervals, and show the spectral evolution in Figure 6. We find clear evidence of cooling (between 770 and $790 \mathrm{~s}$ in Figure 6), while the inferred radius remains essentially constant. This is a clear signature of a thermonuclear burst, and thus of a NS.

We estimated the timescale of this burst using two methods. We fit an exponential model (count rate $\propto e^{-t / \tau_{1}}$ ) to the light curve of the burst after the peak, estimating the timescale $\tau_{1} \approx 16 \pm 1 \mathrm{~s}$. Following Galloway et al. (2008), we estimated an alternative timescale for thermonuclear bursts $\tau_{2}=E_{\text {burst }} / F_{\text {peak }}$, where $E_{\text {burst }}$ is the total fluence during the burst and $F_{\text {peak }}$ is flux at the peak, finding $\tau_{2} \approx 29 \mathrm{~s}$. Galloway et al. (2008) divide bursts into those with $\tau_{2}$ longer than $10 \mathrm{~s}$, and those with $\tau_{2}$ shorter than $10 \mathrm{~s}$. The longer bursts are generally powered by hydrogen burning (with the exception of "giant" bursts involving photospheric radius expansion, which was not seen here), and the short bursts involve only helium burning, since hydrogen burning proceeds more slowly than helium burning (Fujimoto et al. 1981; van Paradijs et al. 1988; Cornelisse et al. 2003). Our measured burst timescale indicates that hydrogen is being accreted, and thus that the donor star is hydrogen-rich, which requires an orbital period $\gtrsim 1.5 \mathrm{hr}$ (e.g., Nelson et al. 1986) and excludes a WD donor.

\subsubsection{Quiescent Behavior}

We used 7 Chandra/ACIS observations taken when all sources were quiescent (Table 1) to study the behavior of Terzan $5 \mathrm{X}-3$ in quiescence before its outburst. We extracted source and background spectra from each observation using CIAO dmextract. We used a combination of a power-law (PEGPWRLW) model and a hydrogen atmosphere for a NS (NSATMOS), with absorption (PHABS) set to our preferred cluster value, the NS radius to $10 \mathrm{~km}$, mass to $1.4 M_{\odot}$, and distance set to $5.9 \mathrm{kpc}$. This model has been previously used to fit its spectrum in one quiescent observation (Heinke et al. 2006b). To study possible spectral variations, we simultaneously fit spectra from each observation in four different Trials, each with different parameters free (Table 3): (I) constraining the NSATMOS and PEGPWRLW components to have the same values between all observations; (II) letting only the power-law normalization vary between observations, while constraining the NSATMOS temperature to be the same; (III) letting only the NSATMOS temperature vary between observations, while constraining the power-law normalization to be the same; (IV) letting both the power-law normalization and the NSATMOS temperature vary between observations. We found no evidence for variation in the power-law photon index $\Gamma$ between observations if we allowed it to vary. Therefore, we tied its value between observations in each Trial (Figure 7).

Trial I gives a poor fit, with $\chi^{2}=116.3$ for 76 D.O.F. An F-test confirms the improvement from allowing the power-law flux to vary (Trial I to II), giving an F-statistic of 6.0 and probability of $4 \times 10^{-5}$ of obtaining such an improvement by chance. Alternatively, allowing the NS temperature to vary (Trial III) also gives an improvement compared to Trial I (F-statistic of 4.2 , chance improvement probability of $1 \times 10^{-3}$ ). Letting both components vary is a substantial improvement compared to allowing the NS temperature alone to vary (comparing III to IV, F-statistic $=2.27$, chance improvement probability of $4.7 \times$ $10^{-2}$ ), while letting both components vary is not preferred over allowing the power-law component alone to vary (comparing II to IV, F-statistic $=0.89$, chance improvement probability 0.50 ). Thus, we identify clear variation in the nonthermal component, but no evidence for variation in the thermal component. 


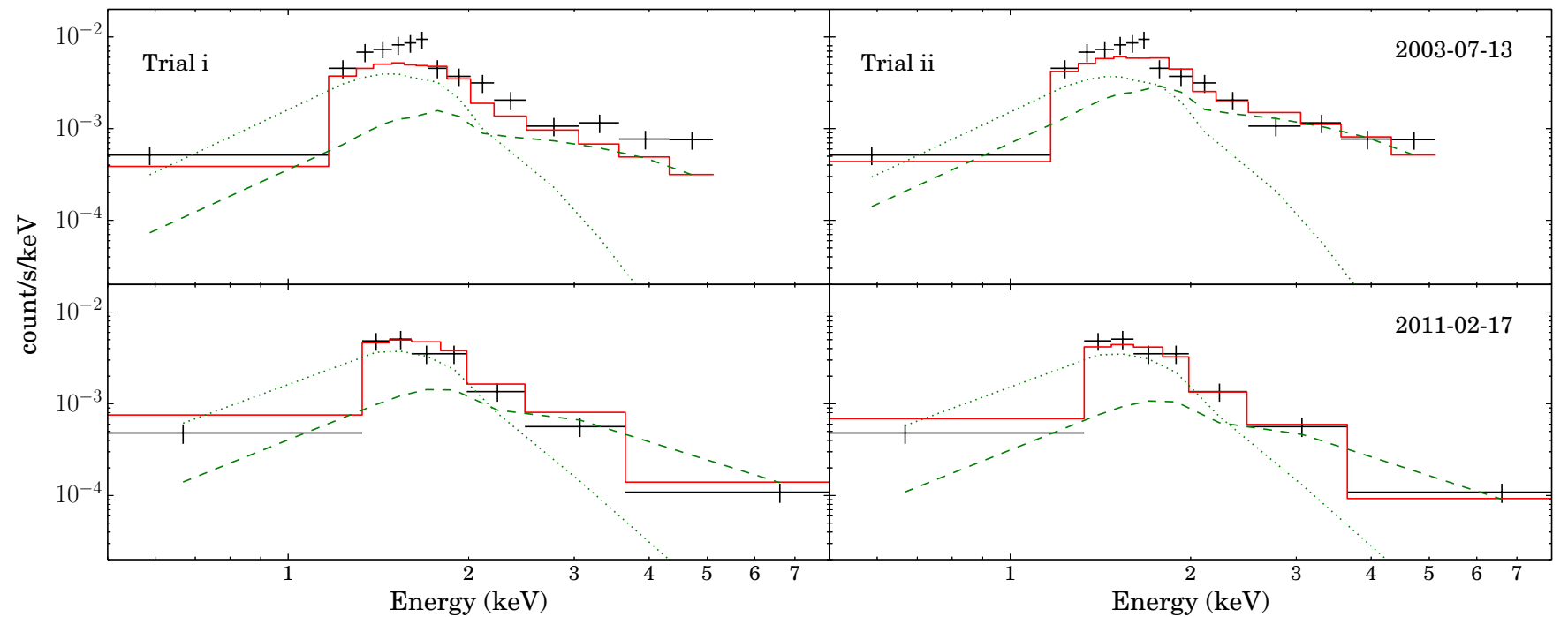

Figure 7. Extracted spectra from two of the Chandra/ACIS observations of Terzan 5 X-3 compared to the fitted model (NSATMOS + PEGPWRLW in XSPEC) from Trials I and II (Table 3). The dotted line indicates the contribution of the NSATMOS, while the dashed line indicates the contribution from the power-law component, and the solid line is their sum. Spectral variations are clear. Comparing Trial II to I, fitting improvement can be seen.

(A color version of this figure is available in the online journal.)

Table 3

Spectral Fitting of 7 Chandra/ACIS Observations of Terzan 5 X-3 During Quiescence (See Table 1), Using PHABS(PEGPWRLW+NSATMOS) in XSPEC

\begin{tabular}{|c|c|c|c|c|c|c|c|}
\hline Trial & ObsID & $\begin{array}{c}\log T \\
(\mathrm{~K})\end{array}$ & $\begin{array}{c}F_{X, \mathrm{NS}}(0.5-10 \mathrm{keV}) \\
\left(10^{-13} \mathrm{erg} \mathrm{cm}^{-2} \mathrm{~s}^{-1}\right)\end{array}$ & $\Gamma$ & $\begin{array}{c}F_{X, \mathrm{PL}}(0.5-10 \mathrm{keV}) \\
\left(10^{-13} \mathrm{erg} \mathrm{cm}^{-2} \mathrm{~s}^{-1}\right)\end{array}$ & $\begin{array}{l}L_{X, \text { total }}(0.5-10 \mathrm{keV}) \\
\left(10^{33} \mathrm{erg} \mathrm{s}^{-1}\right)\end{array}$ & $\chi_{v}^{2} /$ D.O.F.(nhp) \\
\hline I & all & $6.143_{-0.011}^{+0.015}$ & $2.4_{-0.2}^{+0.3}$ & $1.9 \pm 0.4$ & $1.1_{-0.1}^{+0.3}$ & $1.4_{-0.1}^{+0.2}$ & $1.53 / 76(0.002)$ \\
\hline \multirow[t]{7}{*}{ II } & 03798 & $6.138_{-0.018}^{+0.012}$ & $2.4_{-0.5}^{+0.3}$ & $2.1 \pm 0.4$ & $1.9_{-0.4}^{+0.6}$ & $1.8 \pm 0.3$ & $1.09 / 70(0.27)$ \\
\hline & 10059 & $\mathrm{t}$ & $\mathrm{t}$ & $\mathrm{t}$ & $1.3_{-0.3}^{+0.5}$ & $1.5 \pm 0.2$ & $\mathrm{t}$ \\
\hline & 13225 & $\mathrm{t}$ & $\mathrm{t}$ & $\mathrm{t}$ & $0.8_{-0.3}^{+0.4}$ & $1.3 \pm 0.2$ & $\mathrm{t}$ \\
\hline & 13252 & $\mathrm{t}$ & $\mathrm{t}$ & $\mathrm{t}$ & $1.2_{-0.3}^{+0.5}$ & $1.5 \pm 0.2$ & $\mathrm{t}$ \\
\hline & 13705 & $\mathrm{t}$ & $\mathrm{t}$ & $\mathrm{t}$ & $0.7 \pm 0.6$ & $1.3 \pm 0.3$ & $\mathrm{t}$ \\
\hline & 14339 & $\mathrm{t}$ & $\mathrm{t}$ & $\mathrm{t}$ & $0.8_{-0.3}^{+0.5}$ & $1.3 \pm 0.2$ & $\mathrm{t}$ \\
\hline & 13706 & $\mathrm{t}$ & $\mathrm{t}$ & $\mathrm{t}$ & $1.4_{-0.3}^{+0.4}$ & $1.6 \pm 0.2$ & $\mathrm{t}$ \\
\hline \multirow[t]{7}{*}{ III } & 03798 & $6.172_{-0.015}^{+0.011}$ & $3.3_{-0.5}^{+0.4}$ & $1.9 \pm 0.4$ & $1.0_{-0.2}^{+0.3}$ & $1.8 \pm 0.2$ & $1.22 / 70(0.099)$ \\
\hline & 10059 & $6.149_{-0.019}^{+0.014}$ & $2.7_{-0.5}^{+0.3}$ & $\mathrm{t}$ & $\mathrm{t}$ & $1.5 \pm 0.2$ & $\mathrm{t}$ \\
\hline & 13225 & $6.120_{-0.029}^{+0.019}$ & $1.9 \pm 0.5$ & $\mathrm{t}$ & $\mathrm{t}$ & $1.2 \pm 0.2$ & $\mathrm{t}$ \\
\hline & 13252 & $6.144_{-0.019}^{+0.014}$ & $2.4_{-0.5}^{+0.3}$ & $\mathrm{t}$ & $\mathrm{t}$ & $1.4 \pm 0.2$ & $\mathrm{t}$ \\
\hline & 13705 & $6.115_{-0.042}^{+0.025}$ & $1.9_{-0.7}^{+0.5}$ & $\mathrm{t}$ & $\mathrm{t}$ & $1.2 \pm 0.3$ & $\mathrm{t}$ \\
\hline & 14339 & $6.131_{-0.023}^{+0.017}$ & $2.2_{-0.5}^{+0.2}$ & $\mathrm{t}$ & $\mathrm{t}$ & $1.3 \pm 0.2$ & $\mathrm{t}$ \\
\hline & 13706 & $6.155_{-0.017}^{+0.012}$ & $2.7_{-0.5}^{+0.3}$ & $\mathrm{t}$ & $\mathrm{t}$ & $1.5 \pm 0.2$ & $t$ \\
\hline \multirow[t]{7}{*}{$\mathrm{IV}$} & 03798 & $6.161_{-0.021}^{+0.015}$ & $3.0_{-0.6}^{+0.3}$ & $1.7 \pm 0.4$ & $1.5 \pm 0.4$ & $1.9_{-0.3}^{+0.2}$ & $1.10 / 64(0.27)$ \\
\hline & 10059 & $6.148_{-0.021}^{+0.016}$ & $2.7_{-0.5}^{+0.6}$ & $\mathrm{t}$ & $1.1_{-0.3}^{+0.4}$ & $1.6_{-0.2}^{+0.3}$ & $\mathrm{t}$ \\
\hline & 13225 & $6.134_{-0.024}^{+0.015}$ & $2.2_{-0.5}^{+0.2}$ & $\mathrm{t}$ & $0.7_{-0.2}^{+0.3}$ & $1.2 \pm 0.2$ & $\mathrm{t}$ \\
\hline & 13252 & $6.146_{-0.021}^{+0.015}$ & $2.7_{-0.5}^{+0.3}$ & $\mathrm{t}$ & $1.0_{-0.3}^{+0.4}$ & $1.5 \pm 0.2$ & $\mathrm{t}$ \\
\hline & 13705 & $6.121_{-0.043}^{+0.025}$ & $1.7 \pm 1.0$ & $\mathrm{t}$ & $1.3 \pm 0.9$ & $1.2 \pm 0.5$ & $\mathrm{t}$ \\
\hline & 14339 & $6.142_{-0.019}^{+0.014}$ & $2.4_{-0.5}^{+0.3}$ & $\mathrm{t}$ & $0.7_{-0.3}^{+0.4}$ & $1.3 \pm 0.2$ & $\mathrm{t}$ \\
\hline & 13706 & $6.154_{-0.021}^{+0.014}$ & $2.7_{-0.5}^{+0.3}$ & $\mathrm{t}$ & $1.1_{-0.2}^{+0.3}$ & $1.6 \pm 0.2$ & $\mathrm{t}$ \\
\hline
\end{tabular}

Notes. In Trial I both components are constrained to have the same values between observations. In Trials II and III one of the components may vary between observations, while in Trial IV both components are free. We use a " $\mathrm{t}$ " whenever values of a parameter are tied between observations. $k T$ is the NS surface temperature in the star's frame, $\Gamma$ is the power-law photon index, $F_{X, \mathrm{NS}}$ is the unabsorbed flux from the NS atmosphere component, and $F_{X, \mathrm{PL}}$ is the unabsorbed flux from the power-law component. Uncertainties are $90 \%$ confidence intervals. nhp is the null hypothesis probability (otherwise known as the $p$-value).

\subsubsection{Rise of the Outburst}

We fit the Swift/XRT spectra from the rise of the outburst with a two-component model including a thermal component (BBODYRAD in XSPEC) and a nonthermal component (PEGPWRLW in XSPEC). We found good fits permitting only the relative normalizations of the thermal and nonthermal components to vary, with a photon index tied between observations $\left(\Gamma=1.1_{-0.4}^{+0.2}\right)$ and a blackbody radius tied between observations $\left(R=4.3_{-1.2}^{+1.4} \mathrm{~km}\right)$. When the power-law index is left free between observations, the values are consistent, though they 
Table 4

Results of Spectral Analyses for the Rise of the Outburst with a Two-Component Model: Thermal (BBODYRAD in XSPEC) Plus Nonthermal (PEGPWRLW in XSPEC)

\begin{tabular}{|c|c|c|c|c|c|c|c|}
\hline Obs. ID & MJD & $\begin{array}{c}k T \\
(\mathrm{keV})\end{array}$ & $\begin{array}{c}F_{X, \mathrm{BB}}(0.5-10 \mathrm{keV}) \\
\left(10^{-12} \mathrm{erg} \mathrm{s}^{-1} \mathrm{~cm}^{-2}\right)\end{array}$ & $\begin{array}{c}F_{X, \mathrm{PL}}(0.5-10 \mathrm{keV}) \\
\left(10^{-12} \mathrm{erg} \mathrm{s}^{-1} \mathrm{~cm}^{-2}\right)\end{array}$ & $F_{X, \mathrm{PL}} / F_{X, \text { total }}$ & $\begin{array}{c}L_{X, \text { total }}(0.5-10 \mathrm{keV}) \\
\left(10^{34} \mathrm{erg} \mathrm{s}^{-1}\right)\end{array}$ & $\overline{\chi_{v}^{2} / \text { D.O.F. }}$ \\
\hline 91445006 & 56114.8 & $0.31 \pm 0.03$ & $5 \pm 2$ & $5 \pm 2$ & $50 \pm 20 \%$ & $4 \pm 1$ & $0.53 / 6$ \\
\hline 32148003 & 56115.8 & $0.36 \pm 0.03$ & $9 \pm 3$ & $13 \pm 4$ & $59_{-16}^{+15} \%$ & $9 \pm 2$ & $0.68 / 5$ \\
\hline 32148004 & 56117 & $0.41 \pm 0.02$ & $15_{-3}^{+4}$ & $17 \pm 6$ & $53_{-16}^{+12} \%$ & $13 \pm 3$ & $1.19 / 9$ \\
\hline 32148005 & 56118.1 & $0.44_{-0.07}^{+0.05}$ & $20 \pm 10$ & $70 \pm 20$ & $78_{-15}^{+12} \%$ & $37 \pm 9$ & $0.55 / 6$ \\
\hline 32148006 & 56120.7 & $0.67 \pm 0.06$ & $110 \pm 40$ & $500_{-70}^{+60}$ & $82_{-8}^{+7} \%$ & $250 \pm 30$ & $1.39 / 19$ \\
\hline
\end{tabular}

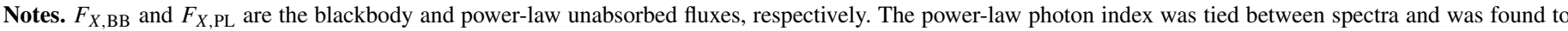

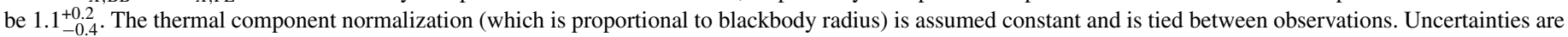
$90 \%$ confidence intervals. $\chi_{v}^{2}$ and degrees of freedom in this table are found by fitting each data set individually based on values found in simultaneous fit.

are poorly constrained in several spectra. Comparing our twocomponent model fits (Table 4) to our power-law fits (Table 2), a clear improvement in the fit is seen. Simultaneous fits to the first five Swift/XRT spectra of the outburst (listed in Table 4) with an absorbed power-law (with the photon index free between observations) give a reduced $\chi^{2}$ of 1.32 for 45 degrees of freedom, while fits with an absorbed power-law plus blackbody give a reduced $\chi^{2}$ of 1.09 for 43 degrees of freedom. An F-test gives an F-statistic of 5.55 and chance improvement probability of 0.007 , supporting the addition of the thermal component. Protassov et al. (2002) showed that the F-test is often inaccurate for testing the necessity of adding an additional spectral component. We therefore chose the spectrum with the clearest evidence of a thermal component (ObsID 32148004), which shows a $\Delta \chi^{2}$ of 6.1 between the power-law and power-law plus thermal spectral fits (going from 12 degrees of freedom to 11). We simulated 1000 data sets using a best-fit absorbed power-law model, and fit them both with a power-law model and with a power-law plus thermal component model. None of our simulations showed a larger $\Delta \chi^{2}$ than that produced by our model, allowing us to conclude that the probability of incorrectly concluding that a thermal component is required is less than $99.5 \%$.

This indicates that the hardening during the outburst rise is likely caused by the decreasing relative contribution of a thermal component. With increasing time, and thus with increasing $L_{x}$, the fractional contribution of the thermal component decreases, but its $k T$ increases monotonically. In the next section, we suggest that the thermal component is due to low-level accretion onto the surface of the NS.

\section{DISCUSSION}

\subsection{Hardening during the Outburst Rise}

We observed clear evidence of hardening of the spectrum during the outburst rise from $L_{X} \sim 4 \times 10^{34}$ up to $10^{36} \mathrm{erg} \mathrm{s}^{-1}$. We have evidence that this hardening is due to the relative reduction in strength of a thermal component in the spectrum with increasing brightness. This is the first time that such hardening during the outburst rise has been detected, made possible by our program of Swift/XRT globular cluster monitoring allowing early detection of the outburst below $L_{X}=10^{35} \mathrm{erg} \mathrm{s}^{-1}$. The trend of inferred photon index (for a fit to a power-law model) versus $L_{X}$ is clear from the data in the rise, and is consistent with the data in the decay (which are not well-sampled below $L_{X}=10^{35} \mathrm{erg} \mathrm{s}^{-1}$ ); see Figure 5 .

Softening during outburst decays has been seen from other (likely) NS LMXBs, in the $L_{X}$ range of $10^{34}-10^{35.5} \mathrm{erg} \mathrm{s}^{-1}$, especially when the soft $(<2 \mathrm{keV}) \mathrm{X}$-ray energy range is included
(Jonker et al. 2003, 2004; Cackett et al. 2011; Fridriksson et al. 2011; Armas Padilla et al. 2011, 2013c). RXTE observations have shown marginal softening during the decay of Aql X-1 down to $L_{X}=5 \times 10^{34} \mathrm{erg} \mathrm{s}^{-1}$ (Maitra \& Bailyn 2004), only in the part of the spectrum below $6 \mathrm{keV}$. RXTE observations of SAX J1808.4-3658 showed almost no spectral changes from $L_{X} \sim 2 \times 10^{36}$ down to $2 \times 10^{34} \mathrm{erg} \mathrm{s}^{-1}$ (Gilfanov et al. 1998). These apparently contrasting observations are consistent if the softening is due to the increasing importance of the thermal component at lower $L_{X}$. Evidence in favor of an increasing relative thermal component can also be seen in Swift/XRT spectra of SAX J1808.4-3658 declining from $L_{X} \sim 10^{36}$ down to $10^{33} \mathrm{erg} \mathrm{s}^{-1}$ (Campana et al. 2008). Thus, we interpret the hardening we observe in Terzan 5 X-3's rise as due primarily to the decreasing importance of a thermal component, rather than to the same physics responsible for the softening of black hole LMXBs during their decay, which show a steepening power-law spectrum (e.g., Plotkin et al. 2013).

Comparing the spectra observed from Terzan 5 X-3 to those of other NS LMXBs, we find a common pattern, that below $L_{X} \sim 1-3 \times 10^{35} \mathrm{erg} \mathrm{s}^{-1}$ a thermal component is often required. For instance, Armas Padilla et al. (2013b) find, using XMMNewton spectra, that two LMXBs at $L_{X}=1-10 \times 10^{34} \mathrm{erg} \mathrm{s}^{-1}$ require a strong thermal component, while this is not critical for another LMXB at $L_{X} \sim 10^{35} \mathrm{erg} \mathrm{s}^{-1}$. Wijnands et al. (2002b), using Chandra find that SAX J1747.0-2853, at $L_{X} \sim 3 \times 10^{35} \mathrm{erg} \mathrm{s}^{-1}$, does not need a thermal component. Armas Padilla et al. (2013c) measure a thermal component to comprise $\sim 20 \%$ of the $0.5-10 \mathrm{keV}$ luminosity for a transient at $L_{X} \sim 9 \times 10^{34} \mathrm{erg} \mathrm{s}^{-1}$ (using XMM), with no evidence (from poorer Swift/XRT spectra) for a thermal component above $L_{X}=2.6 \times 10^{35} \mathrm{erg} \mathrm{s}^{-1}$. Jonker et al. (2003, 2004) study the return to quiescence of XTE J1709-267, finding a thermal component to comprise $\sim 40 \%$ of the flux at $L_{X} \sim$ $4 \times 10^{34} \mathrm{erg} \mathrm{s}^{-1}$, increasing to $>90 \%$ at $2 \times 10^{33} \mathrm{erg} \mathrm{s}^{-1}$. All these results suggest that there is a physical transition operating around $L_{X} \sim 10^{35} \mathrm{erg} \mathrm{s}^{-1}$ which changes the energy spectra.

Such a transition can be provided by the declining optical depth of a hot Comptonizing atmosphere, as seen in numerical calculations of NSs accreting at low rates (Deufel et al. 2001; Popham \& Sunyaev 2001). Deufel et al. 2001 show temperature profiles and emergent spectra for NSs illuminated by high-temperature protons, such as are produced by radiatively inefficient accretion flows. In their Figures 4 and 5 they show that the emergent spectrum is a featureless Comptonized spectrum extending to $\sim 100 \mathrm{keV}$ above $L_{X} \sim 10^{36} \mathrm{erg} \mathrm{s}^{-1}$, which develops a clear $0.5 \mathrm{keV}$ thermal component at $\sim 10^{35} \mathrm{erg} \mathrm{s}^{-1}$, 
Table 5

Orbital Periods, or Other Classification, of the 18 Galactic Globular Cluster LMXBs That Are Persistently Bright or Have Shown Luminous Outbursts Plus Three Quiescent Globular Cluster LMXBs

\begin{tabular}{|c|c|c|c|c|c|c|}
\hline LMXB & Globular Cluster & State & $P_{\text {orb }}$ & Nature & Notes & References \\
\hline $4 \mathrm{U} 1820-30$ & NGC 6624 & $P$ & $11 \mathrm{~min}$ & $\mathrm{U}$ & $\mathrm{X}$ & (1) \\
\hline 4U 0513-40 & NGC 1851 & $P$ & $17 \mathrm{~min}$ & $\mathrm{U}$ & UV & (2) \\
\hline X1850-087 & NGC 6712 & $\mathrm{P}$ & $20.6^{\mathrm{a}} \min$ & $\mathrm{U}$ & UV & (3) \\
\hline M15 X-2 & M 15 & $\mathrm{P}$ & $22.6 \mathrm{~min}$ & $\mathrm{U}$ & UV & (4) \\
\hline NGC 6440 X-2 & NGC 6440 & $\mathrm{~T}$ & $57.3 \mathrm{~min}$ & $\mathrm{U}$ & XP & (5) \\
\hline XB 1832-330 & NGC 6652 & $\mathrm{P}$ & $2.1 \mathrm{hr}$ & $\mathrm{N}$ & $\mathrm{O}$ & (6) \\
\hline 4U 1746-37 & NGC 6441 & $\mathrm{P}$ & $5.16 \mathrm{hr}$ & $\mathrm{N}$ & $\mathrm{X}$ & (7) \\
\hline SAX J1748.9-2021 & NGC 6440 & $\mathrm{~T}$ & $8.7 \mathrm{hr}$ & $\mathrm{N}$ & $\mathrm{XP}$ & (8) \\
\hline IGR J18245-2452 & M28 & $\mathrm{T}$ & $11.0 \mathrm{hr}$ & $\mathrm{N}$ & $\mathrm{XP}$ & (9) \\
\hline GRS $1747-312$ & Terzan 6 & $\mathrm{~T}$ & $12.36 \mathrm{hr}$ & $\mathrm{N}$ & $\mathrm{X}$ & (10) \\
\hline AC 211 & M 15 & $\mathrm{P}$ & $17.1 \mathrm{hr}$ & $\mathrm{N}$ & UV & (11) \\
\hline Terzan 5 X-2 & Terzan 5 & $\mathrm{~T}$ & $21.27 \mathrm{hr}$ & $\mathrm{N}$ & $\mathrm{XP}$ & $(12,13)$ \\
\hline Rapid Burster & Liller 1 & $\mathrm{~T}$ & $?$ & $\mathrm{~N}$ & $\mathrm{~B}$ & (14) \\
\hline EXO 1745-248 & Terzan 5 & $\mathrm{~T}$ & $?$ & $\mathrm{~N}$ & B & (14) \\
\hline Terzan 5 X-3 & Terzan 5 & $\mathrm{~T}$ & $?$ & $\mathrm{~N}$ & B & (15) \\
\hline XB 1732-304 & Terzan 1 & $\mathrm{~T}$ & $?$ & $?$ & $?$ & (16) \\
\hline $4 U 1722-30$ & Terzan 2 & $\mathrm{P}$ & $?$ & $\mathrm{U}$ ? & B & (17) \\
\hline IGR J17361-4441 & NGC 6388 & $\mathrm{~T}$ & $?$ & $?$ & $?$ & (18) \\
\hline \multicolumn{7}{|c|}{ LMXBs with no observed outbursts } \\
\hline 47 Tuc W37 & 47 Tuc & $\mathrm{Q}$ & $3.09 \mathrm{hr}$ & $\mathrm{N}$ & $\mathrm{X}$ & (19) \\
\hline 47 Tuc X5 & 47 Tuc & Q & $8.67 \mathrm{hr}$ & $\mathrm{N}$ & $\mathrm{X}$ & $(20)$ \\
\hline$\omega$ Cen qLMXB & $\omega$ Cen & $\mathrm{Q}$ & $?$ & $\mathrm{~N}$ & $\mathrm{H} \alpha$ & (21) \\
\hline
\end{tabular}

Notes. X-ray bursts have been detected from all the bright LMXBs except for AC 211 in M15 and IGR J17361-4441 in NGC 6388. State: $\mathrm{P}=$ persistent (or active for $>30 \mathrm{yr}$ ), $\mathrm{T}=$ transient, $\mathrm{Q}=$ quiescent (so far). Nature: $\mathrm{U}=$ ultracompact, $\mathrm{N}=$ normal. Notes: $\mathrm{X}=$ period from $\mathrm{X}$-ray photometry, $\mathrm{UV}=$ period from $\mathrm{UV}$ photometry, $\mathrm{XP}=$ period from $\mathrm{X}$-ray pulsations, $\mathrm{O}=$ period from optical photometry, $\mathrm{B}=$ nature of donor inferred from properties of X-ray bursts, $\mathrm{H} \alpha=$ hydrogen seen in optical counterpart spectrum. Notes represent method of measuring $P_{\text {orb }}$ or determining donor nature.

References. (1) Stella et al. 1987; (2) Zurek et al. 2009; (3) Homer et al. 1996; (4) Dieball et al. 2005; (5) Altamirano et al. 2010; (6) Engel et al. 2012; (7) Bałucińska-Church et al. 2004; (8) Altamirano et al. 2008; (9) Papitto et al. 2013; (10) in't Zand et al. 2003; (11) Ilovaisky et al. 1993; (12) Strohmayer et al. 2010; (13) Papitto et al. 2011; (14) Galloway et al. 2008; (15) this work; (16) Guainazzi et al. 1999; (17) in't Zand et al. 2007; (18) Bozzo et al. 2011; (19) Heinke et al. 2005; (20) Heinke et al. 2003 b; (21) Haggard et al. 2004.

${ }^{a}$ Or the alias period of 13 minutes.

and loses the Comptonized tail by $10^{33} \mathrm{erg} \mathrm{s}^{-1}$. This transition is a strikingly accurate match to our observations of Terzan 5 X-3's spectral variations, and to other NS transients discussed in the literature. However calculations of Deufel et al. 2001 underpredict the observed hard power-law components seen in many quiescent LMXBs at low $L_{X}\left(<10^{34} \mathrm{erg} \mathrm{s}^{-1}\right.$, including Terzan 5 X-3 in quiescence). This may arise from their not including the Comptonizing effects of the overlying accretion flow on the observed spectrum. Popham \& Sunyaev (2001) compute solutions for a hot boundary layer, which becomes optically thin for accretion luminosities below $\sim 10^{36} \mathrm{erg} \mathrm{s}^{-1}$, suggesting that some additional Comptonization can be performed by the accretion flow.

The temperature of the thermal component increases monotonically with the total X-ray luminosity during the rise (Table 4), as expected if the thermal component during the rise is produced by accretion. A correlation of thermal component temperature with total luminosity has been suggested from comparisons of multiple sources (Armas Padilla et al. 2013c), but this measurement clearly confirms this correlation in a single source. Furthermore, heating of the NS crust by accretion during the outburst will give rise to a rapidly decaying surface temperature at the end of the outburst (e.g., Cackett et al. 2006b). This effect of an accretion-heated crust could be confused with changing thermal emission from low-level accretion onto the NS surface during the outburst decline, but is not an issue during the outburst rise.

\subsection{Nature of Donors in Globular Cluster X-Ray Binaries}

The detection of a thermonuclear burst during this outburst showed that the accreting object is a NS. Furthermore, the timescale of this thermonuclear burst indicates that the accreted matter contains hydrogen, evidence that the donor is not a white dwarf. With this information, we are now capable of classifying 15 of the 18 known bright Galactic globular cluster LMXBs as either ultracompact $\left(P_{\text {orb }}<1 \mathrm{hr}\right.$, accreting from a hydrogendeficient and/or degenerate donor) or not ultracompact (accreting from a nondegenerate, H-rich star). Five are known to be ultracompact by direct detection of their orbital periods, and seven systems are known not to be ultracompact by direct measurement of their orbital periods. On the basis of X-ray burst behavior indicative of hydrogen burning (Galloway et al. 2008), another three systems can be identified as not ultracompact (Table 5). (4U 1722-30, in Terzan 2, has shown some evidence, by its persistent low-luminosity accretion and burst behavior, in favor of an ultracompact nature; in't Zand et al. 2007.) Thus, the fraction of observed bright globular cluster LMXBs that are ultracompact appears to be between 28 and $44 \%$ (for 5 or 8 of 18). This fraction is believed to be higher than in the rest of the Galaxy (Deutsch et al. 2000), but uncertainties in selection effects mean that we cannot confidently extrapolate the true underlying fraction of ultracompact systems and make clear comparisons to binary population synthesis models (e.g., Ivanova et al. 2008). 


\subsection{Quiescent Counterpart}

We have identified the quiescent counterpart to Terzan 5-X3 with the brightest previously suggested candidate quiescent LMXB in the cluster, CXOGLB J174805.4-244637 (Heinke et al. 2006b). Our spectral analysis reveals evidence for a variable power-law contribution to the quiescent spectrum over timescales of years, but exhibits no evidence for changes to the thermal component. It is fascinating to see clearly here that the quiescent spectral properties appear to lie on a continuum with the outburst properties, with increasing hardening from quiescence, through the early rise, up to the hard state at $L_{X}>10^{36}$ erg s$^{-1}$ (Figure 5).

Chandra quiescent X-ray counterpart searches have now been performed for nine transient cluster LMXBs, of which the three with the faintest outbursts have been identified with very faint $\left(L_{X}<10^{32} \mathrm{erg} \mathrm{s}^{-1}\right)$ quiescent counterparts (M15 X-3, Heinke et al. 2009; NGC 6440 X-2, Heinke et al. 2010; IGR J17361-4441 in NGC 6388, Pooley et al. 2011b), two have spectrally hard counterparts with $L_{X} \sim 10^{32}-10^{33} \mathrm{erg} \mathrm{s}^{-1}$ (EXO 1745-248 in Terzan 5, Wijnands et al. 2005; IGR J18245-2452 in M28, Papitto et al. 2013; Linares et al. 2013), and four have spectrally soft counterparts with $L_{X} \sim$ $10^{32}-10^{33} \mathrm{erg} \mathrm{s}^{-1}$ (SAX J1748.9-2021 in NGC 6440, in't Zand et al. 2001; X1732-304 in Terzan 1, Cackett et al. 2006a; IGR J17480-2446 in Terzan 5, Degenaar \& Wijnands 2011; and Terzan 5 X-3). These identifications support the idea that the faint soft X-ray sources identified as candidate quiescent LMXBs in globular clusters are indeed transient LMXBs, between (relatively bright) outbursts (Heinke et al. 2003c; Wijnands et al. 2013). The brightest of the faint soft $\mathrm{X}$-ray sources should experience relatively high long-term average mass accretion rates, which will cause relatively large amounts of deep crustal heating and keep their cores warm. Such high mass accretion rates suggest frequent outbursts, and thus it is comforting that we have identified the brightest of the faint soft X-ray sources in the clusters NGC 6440, Terzan 5, and Terzan 1 with observed transients. The suggestion that roughly half the quiescent LMXBs in each cluster are easily identifiable in short Chandra observations by showing soft, primarily thermal X-ray spectra and X-ray luminosities between $10^{32}$ and $10^{33} \mathrm{erg} \mathrm{s}^{-1}$ (Heinke et al. 2005) continues to seem reasonable, though it remains unproven.

From the quiescent NS thermal bolometric luminosity $\left(L_{\mathrm{NS},(0.01-10 \mathrm{keV})}=1.5 \times 10^{33} \mathrm{erg} \mathrm{s}^{-1}, k T=118 \mathrm{eV}\right.$ at the surface) of Terzan $5 \mathrm{X}-3$, and its outburst properties, we can make some general statements about its outburst history or neutrino cooling properties, assuming that the quiescent thermal flux is due to heat deposited in the core during multiple accretion episodes (Brown et al. 1998). We estimate the total mass transfer rate onto the NS during this outburst by converting the daily $M A X I / G S C$ 4-10 keV flux estimates (in Crab units) into $0.1-12 \mathrm{keV}$ fluxes (assuming a power-law with photon index set by the nearest Swift/XRT observations), converting the daily Swift/BAT flux estimates into 12-50 keV fluxes (using the same power-law photon index as for the MAXI data), adding these together, and assuming a $1.4 M_{\odot}, 10 \mathrm{~km} \mathrm{NS}$. This gives us a total energy release over the outburst of $9 \times 10^{43} \mathrm{erg}$, and a total mass transfer of $2.4 \times 10^{-10} M_{\odot}$.

If we assume "standard" modified Urca cooling (Yakovlev \& Pethick 2004; Wijnands et al. 2013), then we can estimate (using the quiescent NS luminosity) a mass transfer rate onto the NS of $\dot{M} \sim 3 \times 10^{-11} M_{\odot} \mathrm{yr}^{-1}$ (though this value might vary depending on the choice of crustal composition, e.g., whether a thick light-element layer is present; Page et al. 2004). Assuming this outburst is typical, we derive an average interval of $\sim 8 \mathrm{yr}$ between outbursts. If the average interval between outbursts were much longer than $10 \mathrm{yr}$, then Terzan 5 X-3 would be brighter in quiescence than expected under even the slowest cooling processes. One could attribute its quiescent thermal luminosity to continued accretion, but our analysis of the quiescent observations identifies no evidence for variability in the thermal component, arguing against this explanation. All Terzan 5 X-ray outbursts since 1996 have been identified with their quiescent counterpart with arcsecond precision, except one in 2002 (Wijnands et al. 2002a). The 2002 outburst had an average luminosity of $L_{X} \sim 2 \times 10^{37} \mathrm{erg} \mathrm{s}^{-1}$, peak $L_{X} \sim 4 \times 10^{37} \mathrm{erg} \mathrm{s}^{-1}$, and lasted for $\sim 30$ days (Degenaar \& Wijnands 2012). The 2012 outburst of Terzan 5 X-3 had a similar average luminosity of $L_{X} \sim 2 \times 10^{37} \mathrm{erg} \mathrm{s}^{-1}$, peak $L_{X}=7 \times 10^{37} \mathrm{erg} \mathrm{s}^{-1}$, and lasted for 30 days above $L_{X} \sim 10^{36} \mathrm{erg} \mathrm{s}^{-1}$ (comparable to the RXTE/ASM detection limit for the 2002 outburst). We therefore suggest that the 2002 $\mathrm{X}$-ray outburst is likely to have also been produced by Terzan 5 $\mathrm{X}-3$. This would nicely fit the $\sim 8 \mathrm{yr}$ recurrence time we inferred above.

We thank H. A. Krimm for helpful discussion on the analysis of Swift/BAT survey data, and T. Mihara for helpful discussion on $M A X I / G S C$ calibration issues. We acknowledge financial support from NSERC Discovery Grants (C.O.H., G.R.S.), an Alberta Ingenuity New Faculty Award (C.O.H.) and the Avadh Bhatia Fellowship (J.C.G.). N.D. is supported by NASA through Hubble Postdoctoral Fellowship grant number HSTHF-51287.01-A from the Space Telescope Science Institute. R.W. is supported by an European Research Council Starting Grant. D.A. acknowledges support from the Royal Society. J.H. and D.P. acknowledge support by the National Aeronautics and Space Administration through Chandra Award Number GO2-13045B, issued by the Chandra X-Ray Observatory Center, which is operated by the Smithsonian Astrophysical Observatory for and on behalf of the National Aeronautics Space Administration under contract NAS8-03060.

This research has made use of the following data and software packages: observations made by the Chandra X-Ray Observatory, data obtained from the Chandra Data Archive, software provided by the Chandra X-Ray Center (CXC) in the application package CIAO, MAXI data provided by RIKEN, JAXA and the MAXI team, Swift/BAT transient monitor results provided by the Swift/BAT team, and the Swift/XRT Data Analysis Software (XRTDAS) developed under the responsibility of the ASI Science Data Center (ASDC), Italy. We acknowledge extensive use of the ADS and arXiv.

Facilities: CXO (ACIS), Swift (XRT, BAT), MAXI (GSC)

\section{REFERENCES}

Altamirano, D., Casella, P., Patruno, A., Wijnands, R., \& van der Klis, M. 2008, ApJL, 674, L45

Altamirano, D., Ingram, A., van der Klis, M., et al. 2012a, ApJL, 759, L20

Altamirano, D., Keek, L., Cumming, A., et al. 2012b, MNRAS, 426, 927

Altamirano, D., Patruno, A., Markwardt, C. B., et al. 2010, ApJL, 712, L58

Altamirano, D., Wijnands, R., Heinke, C. O., Sivakoff, G. R., \& Pooley, D. 2012c, ATel, 4264, 1

Anders, E., \& Grevesse, N. 1989, GeCoA, 53, 197

Armas Padilla, M., Degenaar, N., Patruno, A., et al. 2011, MNRAS, 417, 659

Armas Padilla, M., Degenaar, N., Russell, D. M., \& Wijnands, R. 2013a, MNRAS, 428, 3083 
Armas Padilla, M., Degenaar, N., \& Wijnands, R. 2013b, MNRAS, 436, L89 Armas Padilla, M., Wijnands, R., \& Degenaar, N. 2013c, MNRAS, submitted (arXiv:1307.6009)

Arnaud, K. A. 1996, in ASP Conf. Ser. 101, Astronomical Data Analysis Software and Systems V, ed. G. H. Jacoby \& J. Barnes (San Francisco, CA: ASP), 17

Bahramian, A., Heinke, C. O., Sivakoff, G. R., \& Gladstone, J. C. 2013, ApJ, 766,136

Bałucińska-Church, M., Church, M. J., \& Smale, A. P. 2004, MNRAS, 347, 334 Barthelmy, S. D., Barbier, L. M., Cummings, J. R., et al. 2005, SSRv, 120, 143

Blackburn, J. K. 1995, in ASP Conf. Ser. 77, Astronomical Data Analysis Software and Systems IV, ed. R. A. Shaw, H. E. Payne, \& J. J. E. Hayes (San Francisco, CA: ASP), 367

Bordas, P., Kuulkers, E., Alfonso-Garzón, J., et al. 2010, ATel, 2919, 1

Bozzo, E., Ferrigno, C., Stevens, J., et al. 2011, A\&A, 535, L1

Brown, E. F., Bildsten, L., \& Rutledge, R. E. 1998, ApJL, 504, L95

Burrows, D. N., Hill, J. E., Nousek, J. A., et al. 2005, SSRv, 120, 165

Cackett, E. M., Brown, E. F., Miller, J. M., \& Wijnands, R. 2010, ApJ, 720,1325

Cackett, E. M., Fridriksson, J. K., Homan, J., Miller, J. M., \& Wijnands, R. 2011, MNRAS, 414, 3006

Cackett, E. M., Wijnands, R., Heinke, C. O., et al. 2006a, MNRAS, 369, 407

Cackett, E. M., Wijnands, R., Linares, M., et al. 2006b, MNRAS, 372, 479

Campana, S., Colpi, M., Mereghetti, S., Stella, L., \& Tavani, M. 1998, A\&ARv, 8,279

Campana, S., Israel, G. L., Stella, L., Gastaldello, F., \& Mereghetti, S. 2004, ApJ, 601,474

Campana, S., Stella, L., \& Kennea, J. A. 2008, ApJL, 684, L99

Cavecchi, Y., Patruno, A., Haskell, B., et al. 2011, ApJL, 740, L8

Chakraborty, M., Bhattacharyya, S., \& Mukherjee, A. 2011, MNRAS, 418, 490

Corbel, S., Koerding, E., \& Kaaret, P. 2008, MNRAS, 389, 1697

Corbel, S., Tomsick, J. A., \& Kaaret, P. 2006, ApJ, 636, 971

Cornelisse, R., in't Zand, J. J. M., Verbunt, F., et al. 2003, A\&A, 405, 1033

D’Antona, F., Ventura, P., Caloi, V., et al. 2010, ApJL, 715, L63

Degenaar, N., Brown, E. F., \& Wijnands, R. 2011, MNRAS, 418, L152

Degenaar, N., \& Wijnands, R. 2011, MNRAS, 412, L68

Degenaar, N., \& Wijnands, R. 2012, MNRAS, 422, 581

Degenaar, N., Wijnands, R., Brown, E. F., et al. 2013a, ApJ, 775, 48

Degenaar, N., Wijnands, R., \& Miller, J. M. 2013b, ApJL, 767, L31

Deufel, B., Dullemond, C. P., \& Spruit, H. C. 2001, A\&A, 377, 955

Deutsch, E. W., Margon, B., \& Anderson, S. F. 2000, ApJL, 530, L21

Dieball, A., Knigge, C., Zurek, D. R., et al. 2005, ApJL, 634, L105

Done, C., Gierliński, M., \& Kubota, A. 2007, A\&ARv, 15, 1

Engel, M. C., Heinke, C. O., Sivakoff, G. R., Elshamouty, K. G., \& Edmonds, P. D. 2012, ApJ, 747, 119

Esin, A. A., McClintock, J. E., \& Narayan, R. 1997, ApJ, 489, 865

Fender, R. P., Belloni, T. M., \& Gallo, E. 2004, MNRAS, 355, 1105

Ferraro, F. R., Dalessandro, E., Mucciarelli, A., et al. 2009, Natur, 462, 483

Fridriksson, J. K., Homan, J., Wijnands, R., et al. 2011, ApJ, 736, 162

Fruscione, A., McDowell, J. C., Allen, G. E., et al. 2006, Proc. SPIE, $6270,62701 \mathrm{~V}$

Fujimoto, M. Y., Hanawa, T., \& Miyaji, S. 1981, ApJ, 247, 267

Galloway, D. K., Muno, M. P., Hartman, J. M., Psaltis, D., \& Chakrabarty, D. 2008, ApJS, 179, 360

Gardner, E., \& Done, C. 2012, MNRAS, 434, 3454

Gierliński, M., \& Done, C. 2002, MNRAS, 337, 1373

Gilfanov, M., Revnivtsev, M., \& Molkov, S. 2003, A\&A, 410, 217

Gilfanov, M., Revnivtsev, M., Sunyaev, R., \& Churazov, E. 1998, A\&A, 338, L83

Gladstone, J., Done, C., \& Gierliński, M. 2007, MNRAS, 378, 13

Guainazzi, M., Parmar, A. N., \& Oosterbroek, T. 1999, A\&A, 349, 819

Güver, T., \& Özel, F. 2009, MNRAS, 400, 2050

Haggard, D., Cool, A. M., Anderson, J., et al. 2004, ApJ, 613, 512

Harris, W. E. 1996, AJ, 112, 1487

Hasinger, G., \& van der Klis, M. 1989, A\&A, 225, 79

Heinke, C. O., Altamirano, D., Cohn, H. N., et al. 2010, ApJ, 714, 894

Heinke, C. O., Cohn, H. N., \& Lugger, P. M. 2009, ApJ, 692, 584

Heinke, C. O., Edmonds, P. D., Grindlay, J. E., et al. 2003a, ApJ, 590, 809

Heinke, C. O., Grindlay, J. E., \& Edmonds, P. D. 2005, ApJ, 622, 556

Heinke, C. O., Grindlay, J. E., Lloyd, D. A., \& Edmonds, P. D. 2003b, ApJ, 588,452

Heinke, C. O., Grindlay, J. E., Lugger, P. M., et al. 2003c, ApJ, 598, 501

Heinke, C. O., Rybicki, G. B., Narayan, R., \& Grindlay, J. E. 2006a, ApJ, 644, 1090

Heinke, C. O., Wijnands, R., Altamirano, D., Pooley, D., \& Sivakoff, G. R. 2012, ATel, 4249, 1

Heinke, C. O., Wijnands, R., Cohn, H. N., et al. 2006b, ApJ, 651, 1098
Hessels, J. W. T., Ransom, S. M., Stairs, I. H., et al. 2006, Sci, 311, 1901

Homan, J., \& Pooley, D. 2012, ATel, 4302, 1

Homer, L., Charles, P. A., Naylor, T., et al. 1996, MNRAS, 282, L37

Ilovaisky, S. A., Auriere, M., Koch-Miramond, L., et al. 1993, A\&A, 270, 139 in't Zand, J. J. M., Cumming, A., van der Sluys, M. V., Verbunt, F., \& Pols, O. R. 2005, A\&A, 441, 675

in't Zand, J. J. M., Hulleman, F., Markwardt, C. B., et al. 2003, A\&A, 406, 233

in't Zand, J. J. M., Jonker, P. G., \& Markwardt, C. B. 2007, A\&A, 465,953

in't Zand, J. J. M., van Kerkwijk, M. H., Pooley, D., et al. 2001, ApJL, 563, L41 Ivanova, N., Heinke, C. O., Rasio, F. A., Belczynski, K., \& Fregeau, J. M 2008, MNRAS, 386, 553

Jonker, P. G., Galloway, D. K., McClintock, J. E., et al. 2004, MNRAS, 354,666

Jonker, P. G., Méndez, M., Nelemans, G., Wijnands, R., \& van der Klis, M. 2003, MNRAS, 341, 823

Krimm, H. A., Holland, S. T., Corbet, R. H. D., et al. 2013, ApJS, 209, 14

Lanzoni, B., Ferraro, F. R., Dalessandro, E., et al. 2010, ApJ, 717, 653

Lasota, J.-P. 2001, NewAR, 45, 449

Lewin, W. H. G., van Paradijs, J., \& Taam, R. E. 1993, SSRv, 62, 223

Lin, D., Remillard, R. A., \& Homan, J. 2007, ApJ, 667, 1073

Linares, M., Altamirano, D., Chakrabarty, D., Cumming, A., \& Keek, L. 2012, ApJ, 748, 82

Linares, M., Chakrabarty, D., \& van der Klis, M. 2011, ApJL, 733, L17

Linares, M., et al. 2013, MNRAS, in press

Maitra, D., \& Bailyn, C. D. 2004, ApJ, 608, 444

Makishima, K., Ohashi, T., Inoue, H., et al. 1981, ApJL, 247, L23

Massari, D., Mucciarelli, A., Dalessandro, E., et al. 2012, ApJL, 755, L32

Matsuoka, M., Kawasaki, K., Ueno, S., et al. 2009, PASJ, 61, 999

Mihara, T., Nakajima, M., Sugizaki, M., et al. 2011, PASJ, 63, 623

Miller, J. M., Cackett, E. M., \& Reis, R. C. 2009, ApJL, 707, L77

Miller, J. M., Maitra, D., Cackett, E. M., Bhattacharyya, S., \& Strohmayer, T. E. 2011, ApJL, 731, L7

Motta, S., D'A1, A., Papitto, A., et al. 2011, MNRAS, 414, 1508

Nelson, L. A., Rappaport, S. A., \& Joss, P. C. 1986, ApJ, 304, 231

Page, D., Lattimer, J. M., Prakash, M., \& Steiner, A. W. 2004, ApJS, 155, 623

Papitto, A., D’Aì, A., Motta, S., et al. 2011, A\&A, 526, L3

Papitto, A., Di Salvo, T., Burderi, L., et al. 2012, MNRAS, 423, 1178

Papitto, A., Ferrigno, C., Bozzo, E., et al. 2013, Natur, 501, 517

Patruno, A., Bult, P., Gopakumar, A., et al. 2012, ApJL, 746, L27

Peng, F., Brown, E. F., \& Truran, J. W. 2007, ApJ, 654, 1022

Plotkin, R. M., Gallo, E., \& Jonker, P. G. 2013, ApJ, 773, 59

Pooley, D., Homan, J., Altamirano, D., et al. 2011a, ATel, 3743, 1

Pooley, D., Homan, J., Heinke, C., et al. 2010, ATel, 2974, 1

Pooley, D., Homan, J., Heinke, C. O., et al. 2011b, ATel, 3627, 1

Popham, R., \& Sunyaev, R. 2001, ApJ, 547, 355

Protassov, R., van Dyk, D. A., Connors, A., Kashyap, V. L., \& Siemiginowska, A. 2002, ApJ, 571, 545

Pszota, G., Zhang, H., Yuan, F., \& Cui, W. 2008, MNRAS, 389, 423

Rajagopal, M., \& Romani, R. W. 1996, ApJ, 461, 327

Ransom, S. M., Hessels, J. W. T., Stairs, I. H., et al. 2005, Sci, 307, 892

Remillard, R. A., \& McClintock, J. E. 2006, ARA\&A, 44, 49

Riggio, A., Burderi, L., Di Salvo, T., et al. 2012, ApJL, 754, L11

Romano, P., Campana, S., Chincarini, G., et al. 2006, A\&A, 456, 917

Rutledge, R. E., Bildsten, L., Brown, E. F., Pavlov, G. G., \& Zavlin, V. E. 2002, ApJ, 577, 346

Serino, M., Mihara, T., Matsuoka, M., et al. 2012, PASJ, 64, 91

Sivakoff, G. R., Jordán, A., Sarazin, C. L., et al. 2007, ApJ, 660, 1246

Stella, L., Priedhorsky, W., \& White, N. E. 1987, ApJL, 312, L17

Strohmayer, T. E., \& Markwardt, C. B. 2010, ATel, 2929, 1

Strohmayer, T. E., Markwardt, C. B., Pereira, D., \& Smith, E. A. 2010, ATel, 2946, 1

Testa, V., di Salvo, T., D’Antona, F., et al. 2012, A\&A, 547, A28

Titarchuk, L. 1994, ApJ, 434, 570

Valenti, E., Ferraro, F. R., \& Origlia, L. 2007, AJ, 133, 1287

van Paradijs, J., Penninx, W., \& Lewin, W. H. G. 1988, MNRAS, 233, 437

Verbunt, F., Bunk, W., Hasinger, G., \& Johnston, H. M. 1995, A\&A, 300, 732

Verbunt, F., \& Hut, P. 1987, in IAU Symp. 125, The Origin and Evolution of Neutron Stars, ed. D. J. Helfand \& J.-H. Huang (Dordrecht: Reidel), 187

Warwick, R. S., Norton, A. J., Turner, M. J. L., Watson, M. G., \& Willingale, R. 1988, MNRAS, 232, 551

White, N. E., \& Angelini, L. 2001, ApJL, 561, L101

Wijnands, R., Altamirano, D., Heinke, C. O., Sivakoff, G. R., \& Pooley, D. 2012, ATel, 4242, 1

Wijnands, R., Degenaar, N., \& Page, D. 2013, MNRAS, 432, 2366

Wijnands, R., Heinke, C. O., Pooley, D., et al. 2005, ApJ, 618, 883 
Wijnands, R., Homan, J., \& Remillard, R. 2002a, ATel, 101, 1 Wijnands, R., Miller, J. M., \& Wang, Q. D. 2002b, ApJ, 579, 422 Wilms, J., Allen, A., \& McCray, R. 2000, ApJ, 542, 914

Worpel, H., Galloway, D. K., \& Price, D. J. 2013, ApJ, 772, 94

Yakovlev, D. G., \& Pethick, C. J. 2004, ARA\&A, 42, 169
Yuan, F. \& Cui, W. 2005, ApJ, 629, 408

Zampieri, L., Turolla, R., Zane, S., \& Treves, A. 1995, ApJ, 439, 849

Zavlin, V. E., Pavlov, G. G., \& Shibanov, Y. A. 1996, A\&A, 315, 141

Zurek, D. R., Knigge, C., Maccarone, T. J., Dieball, A., \& Long, K. S. 2009, ApJ, 699,1113 